\title{
Contrast transfer and noise considerations in focused-probe electron ptychography
}

Colum M. O’Leary,Gerardo T. Martinez,Emanuela Liberti,Martin J. Humphry,Angus I. Kirkland,Peter D. Nellist
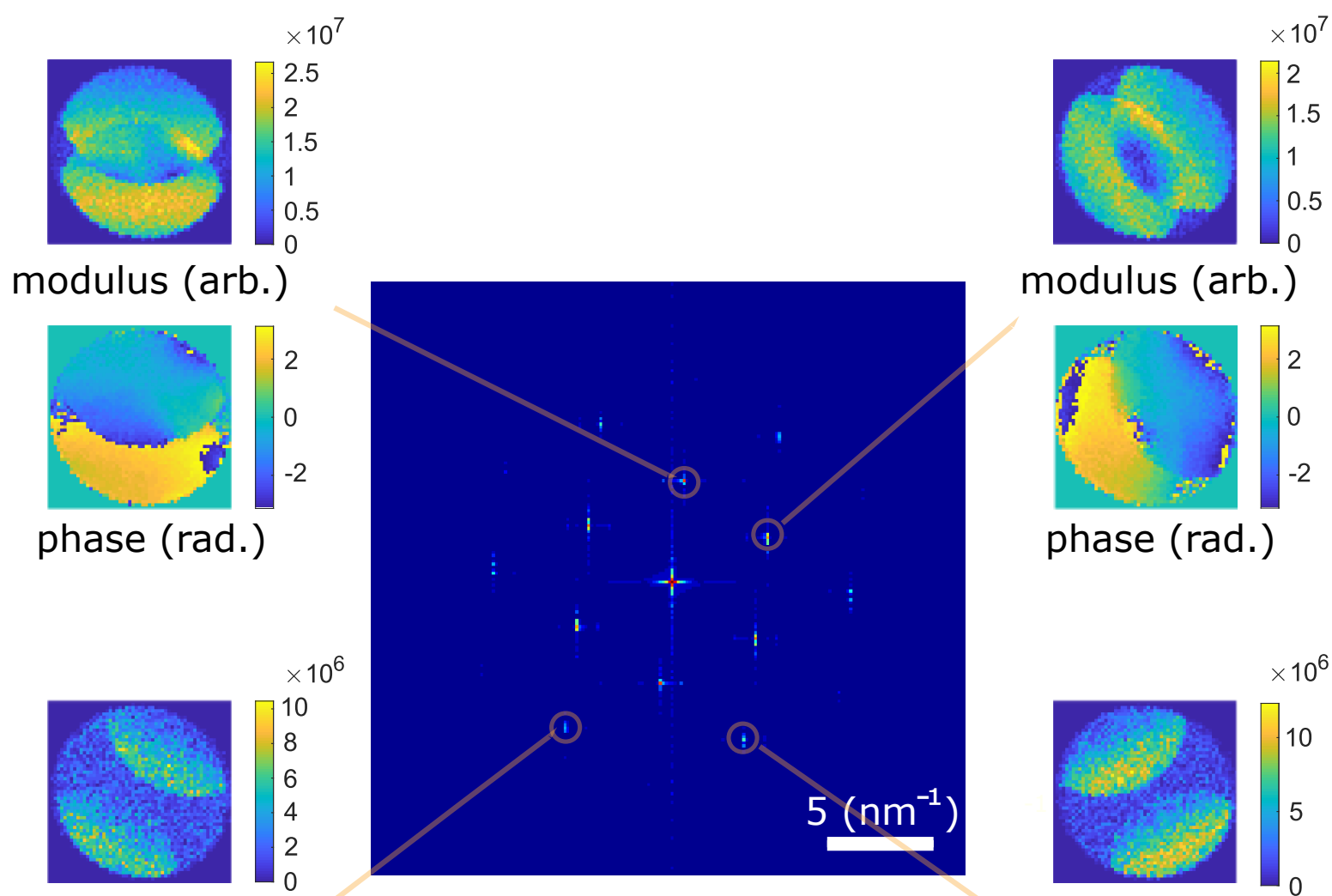

modulus (arb.)

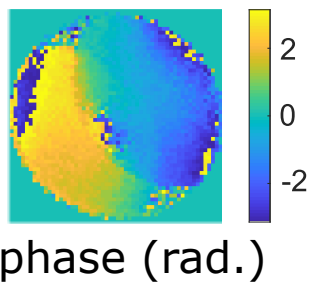

modulus (arb.)

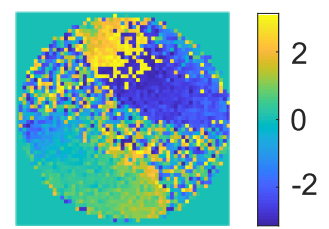

phase (rad.)

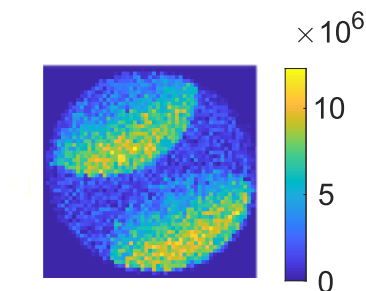

modulus (arb.)

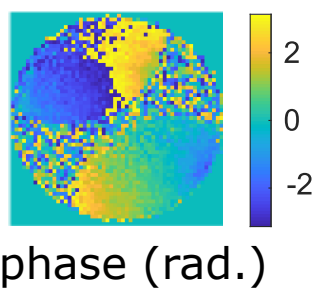


Highlights

\section{Contrast transfer and noise considerations in focused-probe electron ptychography}

Colum M. O’Leary,Gerardo T. Martinez,Emanuela Liberti,Martin J. Humphry,Angus I. Kirkland,Peter D. Nellist

- Contrast transfer in STEM ptychography depends strongly on the convergence angle.

- Target transfer functions are developed to improve ptychographic dose-efficiency.

- The effects of noise on ptychographic reconstructions are discussed. 


\title{
Contrast transfer and noise considerations in focused-probe electron ptychography
}

\author{
Colum M. O’Leary ${ }^{a, *}$, Gerardo T. Martinez ${ }^{a}$, Emanuela Liberti ${ }^{a, b}$, Martin J. Humphry ${ }^{c}$, Angus \\ I. Kirkland ${ }^{a, b, d}$ and Peter D. Nellist ${ }^{a}$ \\ ${ }^{a}$ Department of Materials, University of Oxford, Parks Rd, Oxford OX13PH, United Kingdom. \\ ${ }^{b}$ electron Physical Science Imaging Centre (ePSIC), Diamond Light Source, Didcot OX11 ODE, United Kingdom. \\ ${ }^{c}$ Phase Focus Ltd, Electric Works, Sheffield Digital Campus, Sheffield S1 2BJ, United Kingdom. \\ ${ }^{d}$ The Rosalind Franklin Institute, Harwell Science and Innovation Campus, Didcot OX11 OFA, United Kingdom.
}

\section{ARTICLE INFO}

\section{Keywords:}

Focused-probe electron ptychography

Single side-band method

Contrast transfer function

\begin{abstract}
A B S T R ACT
Electron ptychography is a 4-D STEM phase-contrast imaging technique with applications to lightelement and beam-sensitive materials. Although the electron dose (electrons incident per unit area on the sample) is the primary figure of merit for imaging beam-sensitive materials, it is also necessary to consider the contrast transfer properties of the imaging technique. Here, we explore the contrast transfer properties of electron ptychography. The contrast transfer of focused-probe, non-iterative electron ptychography using the single-side-band (SSB) method is demonstrated experimentally. The band-pass nature of the phase-contrast transfer function (PCTF) for SSB ptychography places strict limitations on the probe convergence semi-angles required to resolve specific sample features with high contrast. The PCTF of the extended ptychographic iterative engine (ePIE) is broader than that for SSB ptychography, although when both high and low spatial frequencies are transferred, band-pass filtering is required to remove image artefacts. Normalisation of the transfer function with respect to the noise level shows that the transfer window is increased while avoiding noise amplification. Avoiding algorithms containing deconvolution steps may also increase the dose-efficiency of ptychographic phase reconstructions.
\end{abstract}

\section{Introduction}

The ability to record images with high contrast at low dose in transmission electron microscopy (TEM) and scanning TEM (STEM) is essential for applications to beamsensitive and light-element materials. Consequently, there has been a substantial effort throughout the microscopy community to establish novel imaging methods which provide directly interpretable contrast while avoiding any specimen damage. The defining figure of merit for such methods is the number of electrons per unit area on the sample; the electron dose. As discussed by Egerton in a recent review [1], minimising electron dose is essential, but it is also crucial to maximise the information obtained from an experiment. The semi-quantitative measure for this is generally expressed as the contrast transfer function (CTF), the theory of which has been well-developed for the majority of conventional TEM and STEM imaging methods.

The CTF is generally defined as the relative information transferred from the sample to the image, at a given spatial frequency $\mathbf{Q}_{\mathbf{p}}$. For conventional TEM imaging, the CTF is highly dependent upon the electron source coherence and objective lens aberrations such as defocus and spherical aberration, as first described by Scherzer in 1949 [2]. Images of samples with varying heights or thicknesses may show contrast reversals, where, for example, the atomic

*Corresponding author

을 cmpoleary93egmail.com (C.M. O’Leary)

ORCID(s): $0000-0002-1899-5320$ (C.M. O'Leary); $0000-0001-5036-0491$ (G.T. Martinez); $0000-0001-5306-7936$ (E. Liberti); $0000-0001-8068-1990$ (A.I. Kirkland); $0000-0002-5883-6463$ (P.D. Nellist) columns of a particular element in a crystal are bright or dark depending on the focus plane with respect to the sample plane. Scherzer demonstrated that, by choosing a specific value of defocus to compensate for the effects of spherical aberration, the contrast reversals could be minimised. This strategy increases the point resolution (spatial frequency at which the first contrast reversal occurs) and thus improves image interpretability. Seventy years on, aberrationcorrected instruments can resolve single atoms, and novel multiple frame imaging and reconstruction methods can provide high-contrast images beyond the point resolution. For example, focal-series and tilt-series methods can provide exit-wave reconstructions with a flat CTF out to and beyond the axial information limit (i.e. the point at which the noise limit makes the signal unrecoverable), respectively $[3,4,5]$. However, obtaining high-resolution exit-wave reconstructions from focal- or tilt-series data is not a trivial task: one requires high instrumental stability, accurate image alignment and careful consideration of CTF contrast reversals and high-order aberrations $[6,7]$.

Bright-field (BF-) STEM imaging shows similar CTFs to those of conventional BF-TEM (as would be expected from the principle of reciprocity [8]), however, there is one drawback. For conventional TEM imaging, a single exposure of the electron wave intensity is acquired using a pixelated detector, e.g. a charge-coupled device (CCD). In order to provide an interpretable image, the signal-to-noise ratio (SNR) must be sufficiently high, and the CTF must be suitably adjusted to correct the lower-order aberrations prior to acquisition. In contrast, conventional STEM imaging methods use 
a single-channel detector to record the integrated intensity of a convergent beam electron diffraction (CBED) pattern at each probe position. Hence, the signal required for each CBED pattern in a two-dimensional scan must be sufficiently strong to provide an image over the entire field of view. Consequently, BF-STEM requires a much larger number of electrons for a given signal-to-noise ratio to provide the same image quality as that for a BF-TEM image.

In 1970, a method of imaging unique to the STEM, known as annular dark-field (ADF-) STEM, was developed by Crewe and co-workers $[9,10]$. The use of an annular detector with a sufficiently large inner angle eliminates diffraction contrast on the image. As a result, with appropriate detector calibration, quantification of ADF images is possible [11]. There is, subsequently, no CTF for ADF-STEM, but there has been defined an optical transfer function (OTF) derived from the Fourier transform of the probe intensity $[12,13]$. ADF-STEM is ideal for the detection of heavy elements in materials, where a considerable amount of highangle electron scattering is collected by the ADF detector. During the development of ADF imaging and the subsequent increase in STEM research, a variety of STEM imaging methods dependent on sample-induced phase shifts were developed. In 1974, Dekkers and de Lang [14] proposed differential phase-contrast (DPC) imaging, whereby a segmented detector is used to calculate the intensity differences between opposite components of the CBED disc such that the electric field of a sample can be mapped. The centre-ofmass (COM) imaging method is based on a similar concept to DPC except that it requires an entire CBED pattern to be recorded at each probe position to relate momentum transfer to the electric field of a sample $[15,16]$. DPC and COM have seen many recent developments to the point that electricfield mapping of single atoms can be achieved [16, 17]. In addition, techniques which integrate over DPC and COM measurements have been developed to produce high-contrast atomic resolution phase images of phase-objects using CTFs which are positive at all $\mathbf{Q}_{\mathbf{p}}[18,19]$. The advantage of such STEM phase-contrast imaging methods is that, unlike TEM imaging, there are no contrast reversals in the CTF. This is particularly advantageous for imaging weak-phase-objects, where in TEM large defoci are required to provide contrast in the image plane [20]. Annular bright-field (ABF) imaging implements a ring-shaped detector as for ADF imaging except using smaller inner and outer collection angles such that, with the correct aberrations, a combination of phase and channelling contrast can provide simultaneous light- and heavy- element imaging. However, the sensitivity of ABF contrast to lens aberrations and sample mis-tilt can make image quantification challenging.

An alternative phase-contrast imaging technique is electron ptychography. Ptychography is not a direct imaging method: reconstruction is performed in silico, i.e. by a postprocessing algorithm. First introduced by Walter Hoppe in 1969 [21, 22, 23], ptychography uses the interference of diffracted beams to reconstruct the sample-induced electron phase shifts. However, unlike most STEM imaging tech- niques, it requires the entire CBED pattern (not just the integrated intensity) at each probe position to produce a phase reconstruction. An array of ptychographic reconstruction algorithms have been developed over the last thirty years, with single-side-band (SSB) [24], Wigner distribution deconvolution (WDD) [25] and the extended ptychographic iterative engine (ePIE) [26] being the most common. More recently, electron ptychography techniques have been used to reconstruct the phase of beam-sensitive materials in the fields of physical and biological sciences. For example, the SSB method has been used to reconstruct a ZSM-5 zeolite sample at 200 e $\AA^{-2}$ [27], while the ePIE method has been used to reconstruct monolayer $\mathrm{MoS}_{2}$ and $\mathrm{WS}_{2}$ at doses below 400 e $\AA^{-2}[28,29,30]$. Furthermore, in an attempt to provide an alternative to cryo-electron microscopy techniques, iterative ptychographic methods such as ePIE have recently been applied to STEM data obtained from biologi-

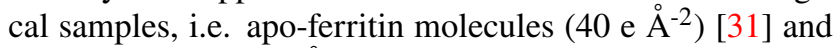
viral particles $\left(5.7\right.$ e $\left.\AA^{-2}\right)$ [20]. The dose-efficiency of ptychography for imaging beam-sensitive materials is strongly dependent on the contrast transfer properties of the algorithm. The CTF for each algorithm should indicate which specific $\mathbf{Q}_{\mathbf{p}}$ values are transferred from the sample plane to the reconstruction, and which $\mathbf{Q}_{\mathbf{p}}$ values are suppressed.

In this paper, the contrast transfer and noise properties of ptychography are explored in order to minimise the electron dose needed to obtain high-quality phase reconstructions. The dependence of the ptychographic CTF on the choice of probe convergence semi-angle and reconstruction algorithm is demonstrated. Furthermore, the existing CTFs for SSB ptychography are modified in order to account for the noise distribution in the data and subsequently increase the ptychographic dose-efficiency.

Section 2 provides a brief introduction to ptychographic techniques, with particular emphasis on SSB and WDD methods. In Section 3, the existing theory of CTFs for SSB ptychography is introduced before measuring the contrast transfer from the phase reconstructions of amorphous and crystalline samples. Section 4 demonstrates the application of a modified transfer function in order to increase the doseefficiency of ptychography. In Section 5, the performance of SSB and WDD ptychography is compared for low-dose experimental data. The conclusions are presented in Section 6.

\section{Electron ptychography techniques}

Although ptychographic imaging is a general technique that is not limited to crystalline samples, it is helpful to first consider samples that lead to discrete diffracted beams. In 4-D STEM, a two-dimensional convergent beam electron diffraction (CBED) pattern is recorded at each probe position in a two-dimensional scan, as shown schematically in Figure 1. For a thin sample which obeys the weak-phaseobject approximation (WPOA), the majority of electrons recorded in the diffraction plane will have been unaffected by the sample, forming the BF disc (denoted 0 in Figure 1). A small proportion of electrons will undergo first-order 
scattering, forming $+\mathrm{g}$ and $-\mathrm{g}$ beams. The interference between the 0 and $+/-\mathrm{g}$ beams is used in ptychography to measure the phase and amplitude of the scattered beams in order to reconstruct the complex transmission function of the sample, $\psi(\mathbf{r})$.

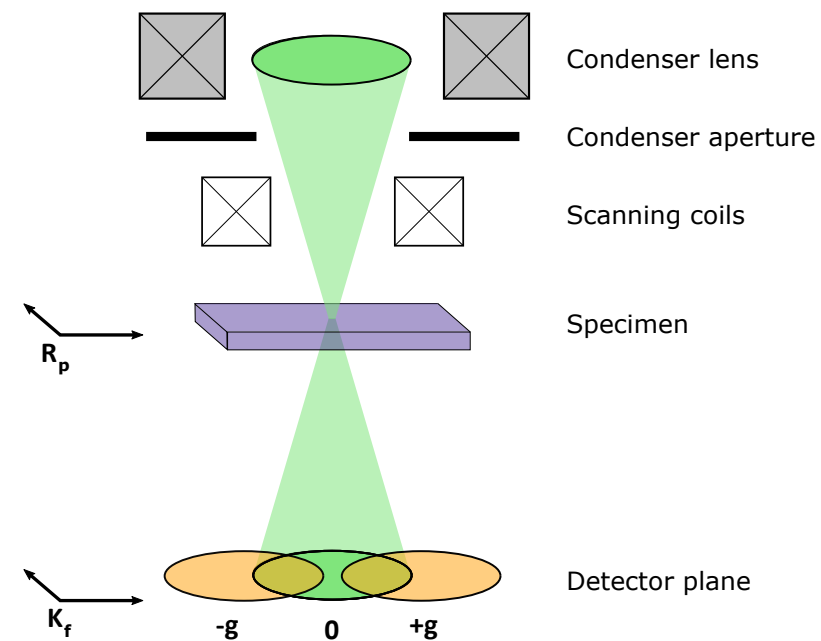

Figure 1: Scanning transmission electron microscopy (STEM) schematic. Following wave transmission through the specimen, a convergent beam electron diffraction (CBED) pattern is formed in the detector plane. The interference between the direct beam and one set of diffracted beams $(+/-\mathrm{g})$ is illustrated for clarity.

Adopting the approach of Rodenburg and Bates [25], the complex amplitude of the matrix of CBED patterns for a $2 \mathrm{D}$ array of probe positions can be expressed as:

$$
M\left(\mathbf{K}_{\mathbf{f}}, \mathbf{R}_{\mathbf{p}}\right)=\left[A\left(\mathbf{K}_{\mathbf{f}}\right) \exp \left(i 2 \pi \mathbf{K}_{\mathbf{f}} \cdot \mathbf{R}_{\mathbf{p}}\right)\right] \otimes_{\mathbf{K}_{\mathbf{f}}} \Psi\left(\mathbf{K}_{\mathbf{f}}\right)
$$

where $\mathbf{K}_{\mathbf{f}}$ and $\mathbf{R}_{\mathbf{p}}$ are the scattering vectors and probe positions respectively, $A\left(\mathbf{K}_{\mathbf{f}}\right)$ is the aperture function and $\Psi\left(\mathbf{K}_{\mathbf{f}}\right)$ is the Fourier transform of the specimen transmission function. There is an important assumption here that the interaction with the sample can be described as a transmission function. The modulus squared, $\left|M\left(\mathbf{K}_{\mathbf{f}}, \mathbf{R}_{\mathbf{p}}\right)\right|^{2}$, is recorded by the detector. The nature of the recording process effectively renders the phase information in the detector plane lost - the so-called phase problem common in X-ray and electron crystallography. Figure 2(a) shows a subset of $\left|\boldsymbol{M}\left(\mathbf{K}_{\mathbf{f}}, \mathbf{R}_{\mathbf{p}}\right)\right|^{2}$ for a ptychographic experiment on a graphene monolayer. The data was acquired using a JEOL 4DCanvas pixelated detector on a JEM-ARM200F at $80 \mathrm{kV}(\alpha=31.5 \mathrm{mrad}$, electron dose $=1.4 \times 10^{6} \mathrm{e}^{-2}$ ). The data for this experiment and those in Sections 3-4 were acquired using four-fold detector binning at 4,000 frames per second. Even at high electron doses, each CBED pattern is dominated by noise. Figure 2(b) shows a subsection of the synthetic BF image intensity with respect to probe position $\mathbf{R}_{\mathbf{p}}$, obtained by summing all $\mathbf{K}_{\mathbf{f}}$ values in the BF disc within an outer collection angle of $0.2 \alpha(6.3 \mathrm{mrad})$. The $\mathrm{BF}$ image provides es- sentially no structural information about the graphene sample, and is dominated by noise. The HAADF image obtained simultaneously with the 4-D STEM data set is shown in Figure 2(c) and reveals the crystalline structure of the monolayer. Imaging with HAADF detectors is typically performed using higher beam currents and faster dwell times $(\sim 10 \mu \mathrm{s})$. Thus, the HAADF signal presented here is similar to what would be expected using conventional HAADF speeds at the same electron dose. However, the relatively slow acquisition is more susceptible to experimental instabilities such as stage drift and scan distortions, and the compensation of these will be the subject of future work. The region of the HAADF image enclosed by the green square is the same region used for the plot of the BF image intensity in Figure 2(b), and is magnified in Figure 2(d). Although the HAADF image provides noticeably greater contrast than the synthetic BF image, it is also dominated by noise. However, by performing dose-efficient techniques on the 4-D STEM data such as focused-probe electron ptychography, high-SNR phase reconstructions can be obtained. The reconstruction process is described below.

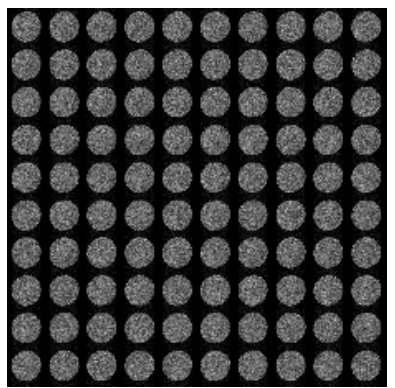

(a)

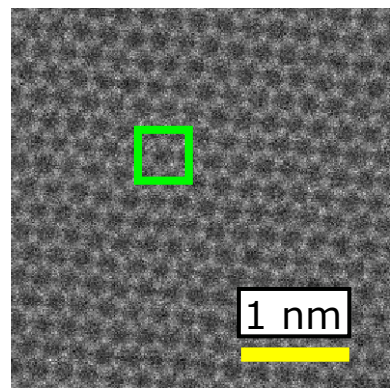

(c)

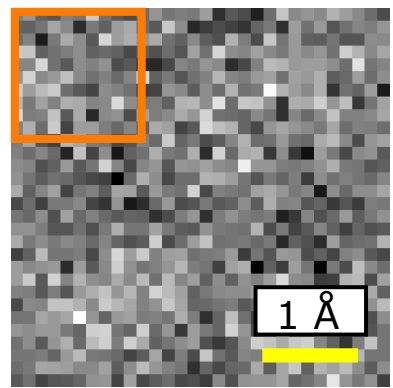

(b)

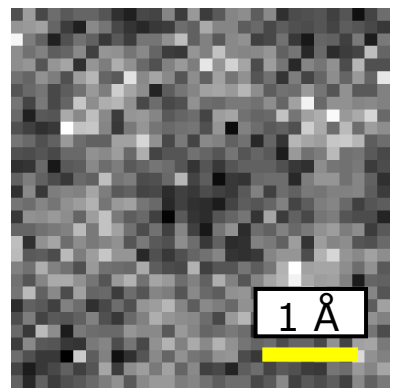

(d)
Figure 2: (a) Subsection of a 4-D STEM data set acquired from a monolayer graphene sample using the JEOL 4DCanvas at $80 \mathrm{kV}$. (b) Synthetic bright-field (BF) image intensity as a function of $\mathbf{R}_{\mathbf{p}}$ obtained by summing all $\mathbf{K}_{\mathbf{f}}$ values within an outer collection angle of $0.2 \alpha(6.3 \mathrm{mrad})$. The CBED patterns displayed in (a) were used to synthesize the region in (b) enclosed by the orange square. (c) HAADF image obtained simultaneously with the 4-D STEM data. The region enclosed by the green square is the same as that plotted in (b), and is magnified in (d).

Performing a Fourier transform of (1) with respect to $\mathbf{R}_{\mathbf{p}}$, 
one obtains:

$$
G\left(\mathbf{K}_{\mathbf{f}}, \mathbf{Q}_{\mathbf{p}}\right)=A\left(\mathbf{K}_{\mathbf{f}}\right) A^{*}\left(\mathbf{K}_{\mathbf{f}}+\mathbf{Q}_{\mathbf{p}}\right) \otimes_{\mathbf{K}_{\mathbf{f}}} \Psi\left(\mathbf{K}_{\mathbf{f}}\right) \Psi^{*}\left(\mathbf{K}_{\mathbf{f}}-\mathbf{Q}_{\mathbf{p}}\right)
$$

where $\mathbf{Q}_{\mathbf{p}}$ is the image spatial frequency - the conjugate variable to probe position. The Fourier transform step effectively 'unlocks' the phase information by separating the specimen function terms from the modulus square. The function $G\left(\mathbf{K}_{\mathbf{f}}, \mathbf{Q}_{\mathbf{p}}\right)$ may be interpreted as a map of the amplitude and phase of the intensity variation in the detector plane at position $\mathbf{K}_{\mathbf{f}}$ and at a spatial frequency with respect to the probe translation of $\mathbf{Q}_{\mathbf{p}}$. Equivalently, one could denote $G\left(\mathbf{K}_{\mathbf{f}}, \mathbf{Q}_{\mathbf{p}}\right)$ as the Fourier transform of an array of 2D STEM images, each recorded for a single detector pixel at $\mathbf{K}_{\mathbf{f}}$. The nature of $G\left(\mathbf{K}_{\mathbf{f}}, \mathbf{Q}_{\mathbf{p}}\right)$ can be illustrated by summing $\left|G\left(\mathbf{K}_{\mathbf{f}}, \mathbf{Q}_{\mathbf{p}}\right)\right|^{2}$ over all $\mathbf{K}_{\mathbf{f}}$ and plotting as a function of $\mathbf{Q}_{\mathbf{p}}$, as displayed in Figure 3. For conventional TEM imaging, the strong regions of diffraction in the back focal plane of the microscope predominantly contribute to the image formation. In contrast, for electron ptychography, the largest values of $G\left(\mathbf{K}_{\mathbf{f}}, \mathbf{Q}_{\mathbf{p}}\right)$ contribute most significantly to the phase reconstruction. Several $\mathbf{Q}_{\mathbf{p}}$ values have been highlighted in Figure 3, for which both the modulus and phase of $G\left(\mathbf{K}_{\mathbf{f}}, \mathbf{Q}_{\mathbf{p}}\right)$ have been plotted as a function of $\mathbf{K}_{\mathbf{f}}$. The function $G\left(\mathbf{K}_{\mathbf{f}}, \mathbf{Q}_{\mathbf{p}}\right)$ is a convolution between the product of a pair of aperture functions separated by $\mathbf{Q}_{\mathbf{p}}$ and the interference between scattered beams separated by $\mathbf{Q}_{\mathbf{p}}$. The aperture function product is non-zero in a disc overlap region, and leads to the features seen in the amplitude and phase shown in Figure 3.

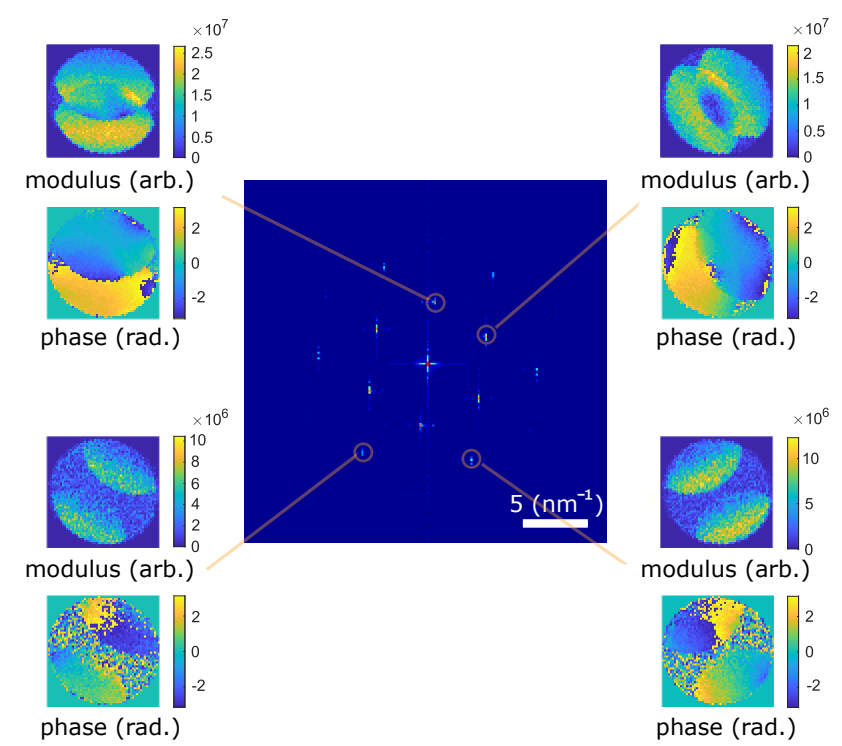

Figure 3: Power spectra of $G\left(\mathbf{K}_{\mathbf{f}}, \mathbf{Q}_{\mathbf{p}}\right)$ with respect to spatial frequency $\mathbf{Q}_{\mathbf{p}}$. For spatial frequencies with large power spectrum values, the modulus and phase of $G\left(\mathbf{K}_{\mathbf{f}}, \mathbf{Q}_{\mathbf{p}}\right)$ have been plotted.

In general, for phase-objects, the specimen information can be retrieved using the Wigner distribution deconvolution (WDD). Firstly, an inverse Fourier transform of $G\left(\mathbf{K}_{\mathbf{f}}, \mathbf{Q}_{\mathbf{p}}\right)$ with respect to $\mathbf{K}_{\mathbf{f}}$ is performed to obtain

$$
H\left(\mathbf{r}, \mathbf{Q}_{\mathbf{p}}\right)=\xi_{P}\left(\mathbf{r},-\mathbf{Q}_{\mathbf{p}}\right) \cdot \xi_{\psi}\left(\mathbf{r}, \mathbf{Q}_{\mathbf{p}}\right)
$$

where $\xi_{P}\left(\mathbf{r},-\mathbf{Q}_{\mathbf{p}}\right)$ and $\xi_{\psi}\left(\mathbf{r}, \mathbf{Q}_{\mathbf{p}}\right)$ represent the Wigner distribution functions (WDF) of the microscope and specimen, respectively. The specimen WDF can subsequently be obtained by applying a Wiener deconvolution:

$$
\xi_{\psi}\left(\mathbf{r}, \mathbf{Q}_{\mathbf{p}}\right)=\frac{\xi_{P}^{*}\left(\mathbf{r},-\mathbf{Q}_{\mathbf{p}}\right) \cdot H\left(\mathbf{r}, \mathbf{Q}_{\mathbf{p}}\right)}{\left|\xi_{P}\left(\mathbf{r},-\mathbf{Q}_{\mathbf{p}}\right)\right|^{2}+\varepsilon}
$$

where $\varepsilon$ is a small term used to prevent division-by-zero errors and minimise noise amplification. Typically, $\varepsilon$ is chosen to be a constant equal to small fraction of $\left|\xi_{P}\left(\mathbf{r},-\mathbf{Q}_{\mathbf{p}}\right)\right|^{2}$. Hence it is convenient to define an epsilon ratio, $\varepsilon_{\text {rat }}$, as:

$$
\varepsilon_{\mathrm{rat}}=\frac{\varepsilon}{\left|\xi_{P}\left(\mathbf{r},-\mathbf{Q}_{\mathbf{p}}\right)\right|^{2}}
$$

Once the deconvolution step has been performed, the complex specimen transmission function, $\psi\left(\mathbf{R}_{\mathbf{p}}\right)$, can be determined. Further details of the WDD algorithm procedure can be found in the existing literature [25, 32]. The WDD method has been applied to light-heavy element structures in recent years since the development of direct electron detectors [33, 34, 35]. However, one of the concerns regarding the WDD method is the division-by-zero error in the deconvolution step. This may result in infinite noise amplification, especially at the low electron doses required for imaging beam-sensitive materials. Here we discuss the single side-band (SSB) method which assumes a WPOA and as such avoids the use of a deconvolution step. It should be noted that, although ptychography is applicable to general phase-objects, a ptychographic transfer function can only be defined for weak-phase-objects. For stronger phase-objects, the phase-contrast is no-longer linearly proportional to the projected potential, due to the non-negligible interference between diffracted beams. As a result, the WPOA breaks down and Equation (2) cannot be simplified further. In this case, transmission cross-coefficients or numerical methods need to be considered to determine the contrast transfer properties of an imaging method [36]. However, for the purpose of this study, the WPOA will be assumed throughout.

In the WPOA, the sample only introduces a small phase change in the electron wave. The transmitted electron wave, $\psi_{w p}(\mathbf{r})$, can be expressed as:

$$
\psi_{w p}(\mathbf{r})=1+i \psi_{s}(\mathbf{r})=1+i \sigma V_{p}(\mathbf{r})
$$


where $\psi_{s}(\mathbf{r})$ is the scattered component of the complex transmission function [37], $V_{p}(\mathbf{r})$ is the projected potential and $\sigma$ is an interaction constant [38]. With this assumption, Equation (2) can then be simplified to a sum of three terms:

$$
\begin{array}{r}
G\left(\mathbf{K}_{\mathbf{f}}, \mathbf{Q}_{\mathbf{p}}\right)=\left|A\left(\mathbf{K}_{\mathbf{f}}\right)\right|^{2} \delta\left(\mathbf{Q}_{\mathbf{p}}\right) \\
+A\left(\mathbf{K}_{\mathbf{f}}\right) A^{*}\left(\mathbf{K}_{\mathbf{f}}+\mathbf{Q}_{\mathbf{p}}\right) \Psi_{s}^{*}\left(-\mathbf{Q}_{\mathbf{p}}\right) \\
+A^{*}\left(\mathbf{K}_{\mathbf{f}}\right) A\left(\mathbf{K}_{\mathbf{f}}-\mathbf{Q}_{\mathbf{p}}\right) \Psi_{s}\left(\mathbf{Q}_{\mathbf{p}}\right)
\end{array}
$$

where $\Psi_{s}\left(\mathbf{K}_{\mathbf{f}}\right)$ represents the scattered component of $\Psi\left(\mathbf{K}_{\mathbf{f}}\right)$. The first term of (7) represents the zero-beam, while the final two terms represent the overlap between the zero-beam and $-\mathbf{Q}_{\mathbf{p}}$ and $+\mathbf{Q}_{\mathbf{p}}$ beams respectively. This is shown schematically in Figure 4 , where $Q_{p}=\left|\mathbf{Q}_{\mathbf{p}}\right|$. Phase information is contained in the double-overlap (DO) regions where only one diffracted beam interferes with the zero-beam. In triple-overlap (TO) regions, i.e. when $Q_{p}<\alpha$, where $\alpha$ is the probe convergence semi-angle, there is overlap between the zero, $-Q_{p}$ and $+Q_{p}$ beams, and destructive interference occurs which, in the absence of aberrations, leads to zero amplitude in $G\left(\mathbf{K}_{\mathbf{f}}, \mathbf{Q}_{\mathbf{p}}\right)$ and a loss of phase information in this region. However, residual aberrations may give rise to nonzero amplitude in the TO region, as is the case for the TO regions shown in Figure 3. When $\alpha<Q_{p}<2 \alpha$, the $-Q_{p}$ and $+Q_{p}$ beams no longer overlap with each other and the TO region disappears. At $Q_{p}>2 \alpha$, none of the beams overlap and no phase information is transferred. For each $\mathbf{Q}_{\mathbf{p}}$ value, the area of overlap between the zero-beams and diffracted beams is directly proportional to the signal strength. The effects of this on the ptychographic phase reconstruction will be discussed in Sections 3 and 4, but it is emphasised that the function $G\left(\mathbf{K}_{\mathbf{f}}, \mathbf{Q}_{\mathbf{p}}\right)$ is general to 4-D STEM and encodes the information content used by any 4-D STEM phase imaging technique including COM [16], integrated COM (iCOM) [18] and DPC imaging [17]. The integrals over the detector plane performed in these techniques, or indeed the implicit integral performed by a segmented detector, can all be applied to $G\left(\mathbf{K}_{\mathbf{f}}, \mathbf{Q}_{\mathbf{p}}\right)$ to reveal how the information is transferred for a given image spatial frequency, $\mathbf{Q}_{\mathbf{p}}$.

Although aberration-corrected instruments have enabled atomic-resolution capabilities of (S)TEM imaging techniques, it is still often necessary to manually fine-tune the aberrations before acquisition. This requires exposing a sample region of interest to the electron beam: a process which can cause damage to beam-sensitive materials. One of the novel capabilities of ptychographic techniques is the determination of residual aberrations from $G\left(\mathbf{K}_{\mathbf{f}}, \mathbf{Q}_{\mathbf{p}}\right)$ using post-processing matrix inversion techniques such as singular value decomposition. This has previously been demonstrated by Yang et al. for the WDD method: using the determined residual aberrations, a pair of aberrated probe functions was synthesized and used in the deconvolution step of the algorithm, such that the resultant complex transmission function was effectively aberration-corrected
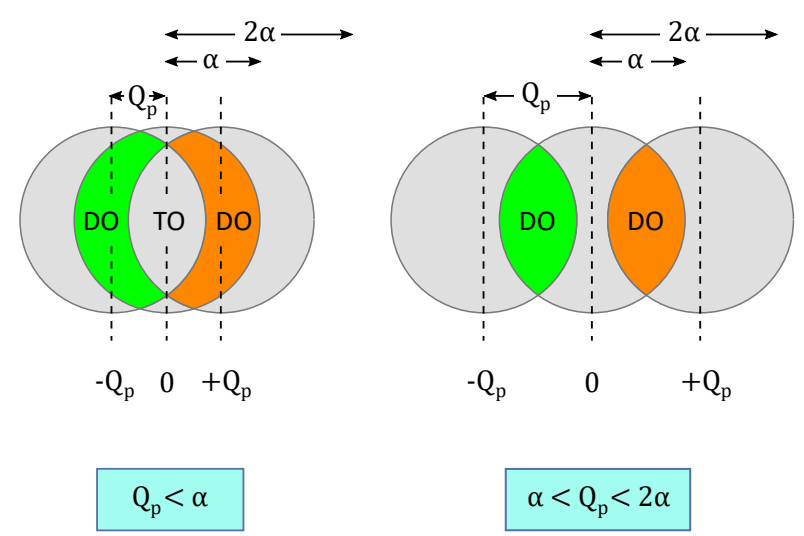

$\alpha<\mathrm{Q}_{\mathrm{p}}<2 \alpha$

Figure 4: Schematic of the phase of $G\left(\mathbf{K}_{\mathrm{f}}, \mathbf{Q}_{\mathbf{p}}\right)$ for spatial frequencies where (a) $Q_{p}<\alpha$, and (b) $\alpha<Q_{p}<2 \alpha$. Regions labelled DO and TO represent the double- and triple-overlap regions, respectively.

[33]. This removes the need to manually fine-tune aberrations and thus reduces the total electron dose used in experiments. Aberration correction can also be performed using the SSB method. In previous work, the aperture aberration function is ignored in the SSB reconstruction process $[37,39]$. However, by multiplying the $-\mathbf{Q}_{\mathbf{p}}$ and $+\mathbf{Q}_{\mathbf{p}}$ interference terms in (7) by $A^{*}\left(\mathbf{K}_{\mathbf{f}}\right) A\left(\mathbf{K}_{\mathbf{f}}+\mathbf{Q}_{\mathbf{p}}\right)$ and $A\left(\mathbf{K}_{\mathbf{f}}\right) A^{*}\left(\mathbf{K}_{\mathbf{f}}-\mathbf{Q}_{\mathbf{p}}\right)$ respectively, the aberrations can be corrected before reconstructing the ptychographic phase. Aberration-corrected SSB ptychography was performed for the atomic-resolution reconstructions presented in Section 3 and Section 4.

Retrieving the exit-wave from (7) consists of three steps. Firstly, the amplitude and phase of either the $-\mathbf{Q}_{\mathbf{p}}$ or $+\mathbf{Q}_{\mathbf{p}}$ DO region in (7) (i.e. one side-band) are summed over all $\mathbf{K}_{\mathbf{f}}$ for each value of $\mathbf{Q}_{\mathbf{p}}$ to obtain the Fourier transmission function $\Psi_{s}\left(\mathbf{Q}_{\mathbf{p}}\right)$. Secondly, an inverse Fourier transform is performed to obtain the real-space complex transmission function, $\psi_{s}\left(\mathbf{R}_{\mathbf{p}}\right)$. Finally, the complex component of $\psi_{s}\left(\mathbf{R}_{\mathbf{p}}\right)$ can be plotted for each probe position in the STEM scan to produce a ptychographic phase reconstruction. Figure 5(a) displays the SSB phase reconstruction from the same graphene monolayer region as shown for the HAADF image in Figure 2(c). The region enclosed by the green square is the same as that shown in Figure 2(c), and is magnified in Figure 5(b). The contrast of the ptychographic reconstructions is significantly greater than that for the equivalent $\mathrm{BF}$ and HAADF images in Figure 2, and demonstrates the high doseefficiency of electron ptychography.

Both SSB and WDD ptychography are non-iterative methods - they involve only Fourier transforms and deconvolutions. There are, however, iterative algorithms, such as the extended ptychographical iterative engine (ePIE), which can reconstruct the complex transmission function of phase-objects. Starting from initial guesses for the probe and object functions, a series of forward calculations is performed to determine the probe and exit-wave [26, 40]. The ePIE method is particularly effective using defocused- 

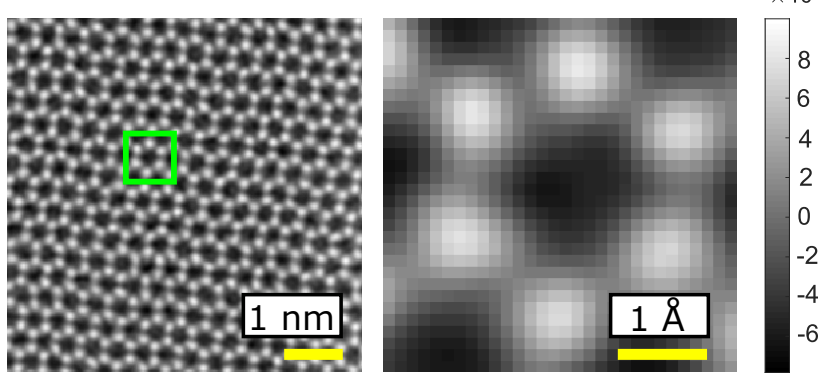

Figure 5: (a) Single side-band (SSB) phase reconstruction of a graphene monolayer from the same region shown in Figure 2(c). The region enclosed by the green square is magnified in (b). Greyscale units: radians.

probe data with significant overlap between adjacent probe positions [41, 28].

\section{Contrast transfer in SSB ptychography}

The area of the DO regions described in Section 2 and illustrated in Figure 4 are the domains in the STEM detector plane in which information about a WPO is recorded. Other regions in the detector plane can be regarded as noise. The area of the DO domains can be plotted as a function of spatial frequency, $\mathbf{Q}_{\mathbf{p}}$, to determine a phase-contrast transfer function (PCTF) intrinsic to SSB ptychography. As derived geometrically by Yang et al. [42], the intrinsic PCTF for SSB ptychography is given by

$$
\begin{aligned}
\operatorname{PCTF}(\omega) & =\operatorname{Re}\left\{\frac { 2 } { \pi } \left[\cos ^{-1}\left(\frac{\omega}{2}\right)-\cos ^{-1}(\omega)\right.\right. \\
& \left.\left.+\omega \sqrt{1-\omega^{2}}-\frac{\omega}{2} \sqrt{1-\left(\frac{\omega}{2}\right)^{2}}\right]\right\}
\end{aligned}
$$

where $\omega=Q_{p} / \alpha$, and $\alpha$ is the probe convergence semiangle. For simplicity, the spatial frequency $Q_{p}$ is expressed in units of angle (mrad) rather than reciprocal distance.

Alternatively, the PCTF can be defined as a function of shifted aperture functions [43]. Using the same nomenclature as in Section 2, the PCTF can be written as:

$$
\operatorname{PCTF}\left(Q_{p}\right)=\frac{\sum_{\mathbf{K}_{\mathbf{f}}}\left|\zeta\left(\mathbf{K}_{\mathbf{f}}, \mathbf{Q}_{\mathbf{p}}\right)\right|}{2 \sum_{\mathbf{K}_{\mathbf{f}}}\left|A\left(\mathbf{K}_{\mathbf{f}}\right)\right|}
$$

where

$$
\zeta\left(\mathbf{K}_{\mathbf{f}}, \mathbf{Q}_{\mathbf{p}}\right)=A^{*}\left(\mathbf{K}_{\mathbf{f}}\right) A\left(\mathbf{K}_{\mathbf{f}}-\mathbf{Q}_{\mathbf{p}}\right)-A\left(\mathbf{K}_{\mathbf{f}}\right) A^{*}\left(\mathbf{K}_{\mathbf{f}}+\mathbf{Q}_{\mathbf{p}}\right)
$$

The PCTF described by (8) (and by (9) in the absence of aberrations) is plotted in Figure 6. The shape of $G\left(\mathbf{K}_{\mathbf{f}}, \mathbf{Q}_{\mathbf{p}}\right)$ is illustrated for $\omega=0.25,0.75,1.25$ and 1.75 respectively. The two most noticeable features of this plot are the range for which the PCTF is non-zero, and the roll-off of low and high $G\left(\mathbf{K}_{\mathbf{f}}, \mathbf{Q}_{\mathbf{p}}\right)$ values, respectively.

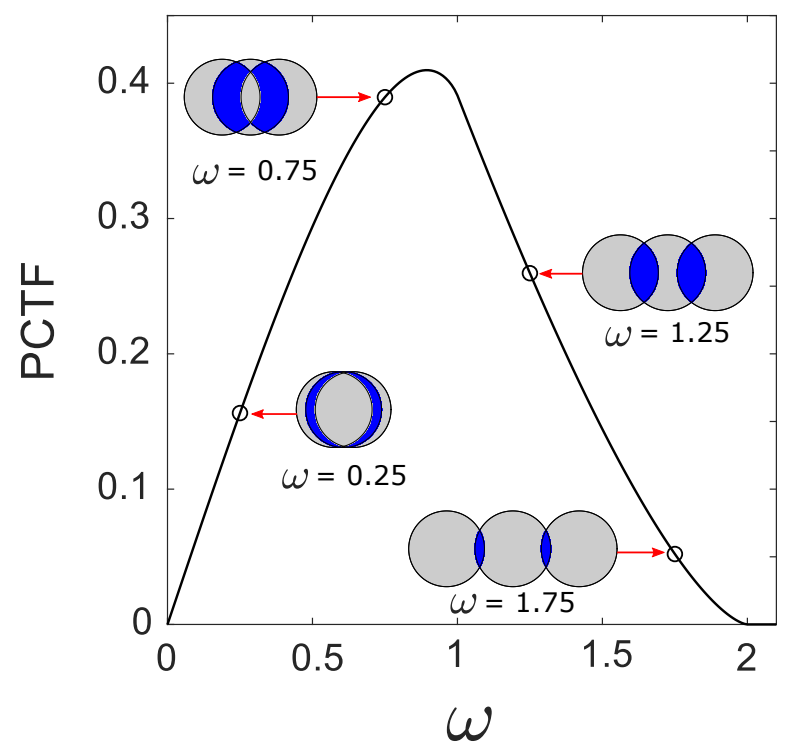

Figure 6: Phase-contrast transfer function (PCTF) for SSB ptychography. The shape of $G\left(\mathbf{K}_{\mathbf{f}}, \mathbf{Q}_{\mathbf{p}}\right)$ has been plotted for $\omega$ $=0.25,0.75,1.25$ and 1.75 .

The effects of (8) and (9) can be measured by applying ptychography to an amorphous sample. Amorphous specimens possess a continuous range of spatial frequencies throughout an aperture-limited diffraction space. In this case, a region of $20 \mathrm{~nm}$-thick amorphous carbon was used. The 4-D STEM data was acquired with the 4DCanvas at $80 \mathrm{kV}$ using two different convergence semi-angles: 7.5 $\mathrm{mrad}$ and $14.4 \mathrm{mrad}$, with cumulative electron doses of $3.3 \times$ $10^{5}$ e $\AA^{-2}$ and $1.6 \times 10^{5}$ e $\AA^{-2}$, respectively. Example values of $G\left(\mathbf{K}_{\mathbf{f}}, \mathbf{Q}_{\mathbf{p}}\right)$ for $\left|\mathbf{Q}_{\mathbf{p}}\right|=6 \mathrm{mrad}$ shown in Figure 7 clearly demonstrate the applicability of ptychography to amorphous materials, as first shown by Rodenburg and co-workers [24].

Following ptychographic reconstruction of the 4-D STEM data, the Fourier transform of the reconstructed electron phase was calculated, the results of which are shown in Figure 8(a)-(b), before computing a radial average to determine the contrast transfer. This is the ptychography equivalent to the Thon ring analysis in HRTEM [44]. Strictly, the resultant plot, $\gamma\left(Q_{p}\right)$, is the PCTF multiplied by the scattering factor at each spatial frequency, i.e.

$$
\gamma\left(Q_{p}\right)=\operatorname{PCTF}\left(Q_{p}\right) \times \operatorname{RA}\left\{F T\left[V_{p}\left(\mathbf{R}_{\mathbf{p}}\right)\right]\right\}
$$

where RA denotes a radial average and $V_{p}\left(\mathbf{R}_{\mathbf{p}}\right)$ is the projected potential at each probe position. Figure 8 shows plots of (c) $\gamma\left(Q_{p}\right)$ and (d) $\gamma(\omega)$ for $7.5 \mathrm{mrad}$ (solid lines) and 14.4 mrad (dashed lines). The relationship between the choice of aperture and the transfer of $Q_{p}$ values into the reconstruction is obvious, and the shape of $\gamma(\omega)$ is maintained regardless of aperture width. To calculate the true experimental PCTF, the projected potentials of the amorphous carbon 


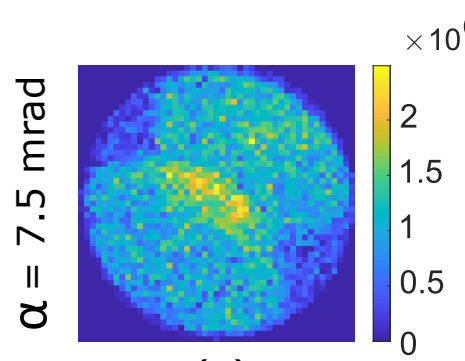

(a)

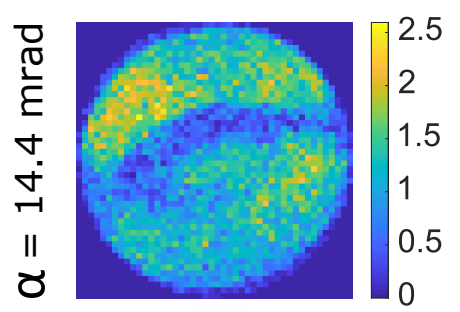

(c)

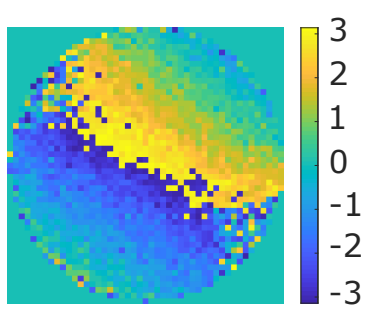

(b)

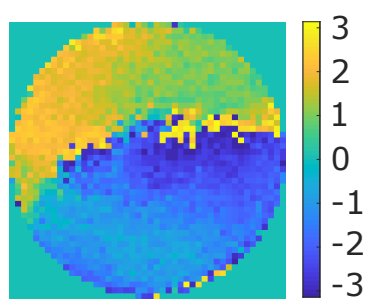

(d)
Figure 7: (a),(c) Modulus (arb. units) and (b),(d) phase (radians) of $G\left(\mathbf{K}_{\mathbf{f}}, \mathbf{Q}_{\mathbf{p}}\right)$ for amorphous carbon data sets at an example spatial frequency, $\left|\mathbf{Q}_{\mathbf{p}}\right|=6 \mathrm{mrad}$, using probe convergence semi-angles of (a)-(b) $14.4 \mathrm{mrad}$ and (c)-(d) $7.5 \mathrm{mrad}$.

would need to be simulated and divided from the radial average of $F T\left[V_{p}\left(\mathbf{R}_{\mathbf{p}}\right)\right]$ to obtain $\operatorname{PCTF}\left(Q_{p}\right)$. However, for the purposes of this study, $\gamma\left(Q_{p}\right)$ suffices to demonstrate the inherent decay of transfer at both low and high spatial frequencies.

When performing STEM imaging and diffraction studies on crystalline samples, it is essential to choose a probeforming aperture which transfers $\mathbf{Q}_{\mathbf{p}}$ values that correspond to the important length scales in the sample with maximum contrast. This is well-known to the electron microscopist, and has recently been demonstrated for 4-D STEM using numerical probe intensity calculations [45]. However, for phase reconstruction techniques such as ptychography, it is particularly important to consider how the PCTF changes with aperture diameter. This dependence will determine which $\mathbf{Q}_{\mathbf{p}}$ values are located at the centre of the passband and transferred to the reconstruction with high contrast, and which $\mathbf{Q}_{\mathbf{p}}$ values are located at the edges of the passband and subsequently suppressed (see Figure 6). As an example we consider a sample containing reflections at discrete spatial frequencies, such as in a graphene monolayer. If a similar 4-D STEM convergence semi-angle series is acquired as for the amorphous carbon experiment and the phase is reconstructed using electron ptychography, the effects of the PCTF can be visualised more clearly. The PCTF for each convergence semi-angle was calculated using (8) and is displayed in Figure 9(a). The right-hand-side of Figure 9 displays the SSB ptychographic reconstruction of graphene data sets for $\alpha=$ (b) $31.5 \mathrm{mrad}$, (c) $22.5 \mathrm{mrad}$, (d) $14.4 \mathrm{mrad}$ and (e) $7.5 \mathrm{mrad}$. The microscope emission current was constant $(11 \mu \mathrm{A})$, such that the electron doses in Figure 9(b) (e) were $2.3 \times 10^{6} \mathrm{e}^{-2}, 1.2 \times 10^{6} \mathrm{e}^{-2}, 5.4 \times 10^{5} \mathrm{e}^{-2}$ and $2.5 \times 10^{5}$ e $\AA^{-2}$, respectively. For the case of the phase reconstructions shown in Figure 9(b) - (c), the aberrations were corrected during the reconstruction process. The reciprocal space locations of the reflections corresponding to the $\{1 \overline{1} 00\}$ (blue line) and $\{2 \overline{1} \overline{1} 0\}$ (red line) lattice vectors of graphene are also shown. Transfer of the $\{1 \overline{1} 00\}$ reflections is required to visualise the hexagons, while transfer of both $\{1 \overline{1} 00\}$ and $\{2 \overline{1} \overline{1} 0\}$ reflections is required to obtain an atomic-resolution reconstruction. For $\alpha=31.5 \mathrm{mrad}$, single atoms are well resolved and the PCTF transfers both the $\{1 \overline{1} 00\}$ and $\{2 \overline{1} \overline{1} 0\}$ reflections. Decreasing the convergence angle to $22.5 \mathrm{mrad}$, atomic resolution is still achieved (both the $\{1 \overline{1} 00\}$ and $\{2 \overline{1} \overline{1} 0\}$ reflections are resolved), but the contrast is diminished due to the position of the $\{2 \overline{1} \overline{1} 0\}$ reflections with respect to the PCTF. The achievable resolution is decreased, and the noise at higher $\mathbf{Q}_{\mathbf{p}}$ values decreases the contrast. At $\alpha=14.4 \mathrm{mrad}$, only the $\{1 \overline{1} 00\}$ reflections can be resolved as the second ring lies outside of the aperturelimited PCTF, while data recorded with $\alpha=7.5 \mathrm{mrad}$ resolves neither family of reflections. In summary, to maximise information for a given electron dose, a probe-forming aperture must be chosen such that all the spatial frequencies of interest in the sample are transferred strongly which is equivalent to their DO regions in $G\left(\mathbf{K}_{\mathbf{f}}, \mathbf{Q}_{\mathbf{p}}\right)$ being as large as possible. As stated earlier, this criterion also applies to other phase detection techniques such as COM, iCOM and DPC.

It should also be noted that, for lower acceleration voltages (i.e. $\leq 60 \mathrm{kV}$ ), the effects of chromatic aberration decrease the area of the DO regions in $G\left(\mathbf{K}_{\mathbf{f}}, \mathbf{Q}_{\mathbf{p}}\right)$ that contain useful sample information [37, 39]. By choosing only the pixels which lie on the 'achromatic lines' in each DO region to perform the SSB reconstruction, the effects of chromatic aberration can be avoided at the expense of signal strength and dose-efficiency [24, 37]. At higher acceleration voltages, such as those used for the experiments shown in this manuscript, these effects can be neglected.

\section{Effects of PCTFs, target PCTFs and noise normalisation}

In addition to choosing the optimum aperture for a given sample, there are still challenges in optimising contrast for ptychography. In SSB ptychography, the PCTF acts as an effective band-pass filter, suppressing both low and high spatial frequencies, as shown in Figure 10. This enables the selection of information that is transferred to the image plane, at the expense of lost information at other spatial frequencies. To compare the transfer properties of SSB ptychography to iterative methods, the PCTF of the ePIE method was calculated for isolated atom simulations $\left(\mathrm{V}_{\mathrm{acc}}=200\right.$ $\mathrm{kV}, \alpha=22.48 \mathrm{mrad})$ using the simulation package, MULTEM [46]. By deconvolving the potential from the ptychographic phase for each atom via (10), the PCTFs could be determined, as shown in Figure 10. In contrast to the SSB method, ePIE converges to a solution for $\psi(\mathbf{r})$ which transfers almost all spatial frequencies with equal weight, before decaying to zero at spatial frequencies beyond $2 \alpha$. To illus- 
Contrast transfer and noise considerations in focused-probe electron ptychography
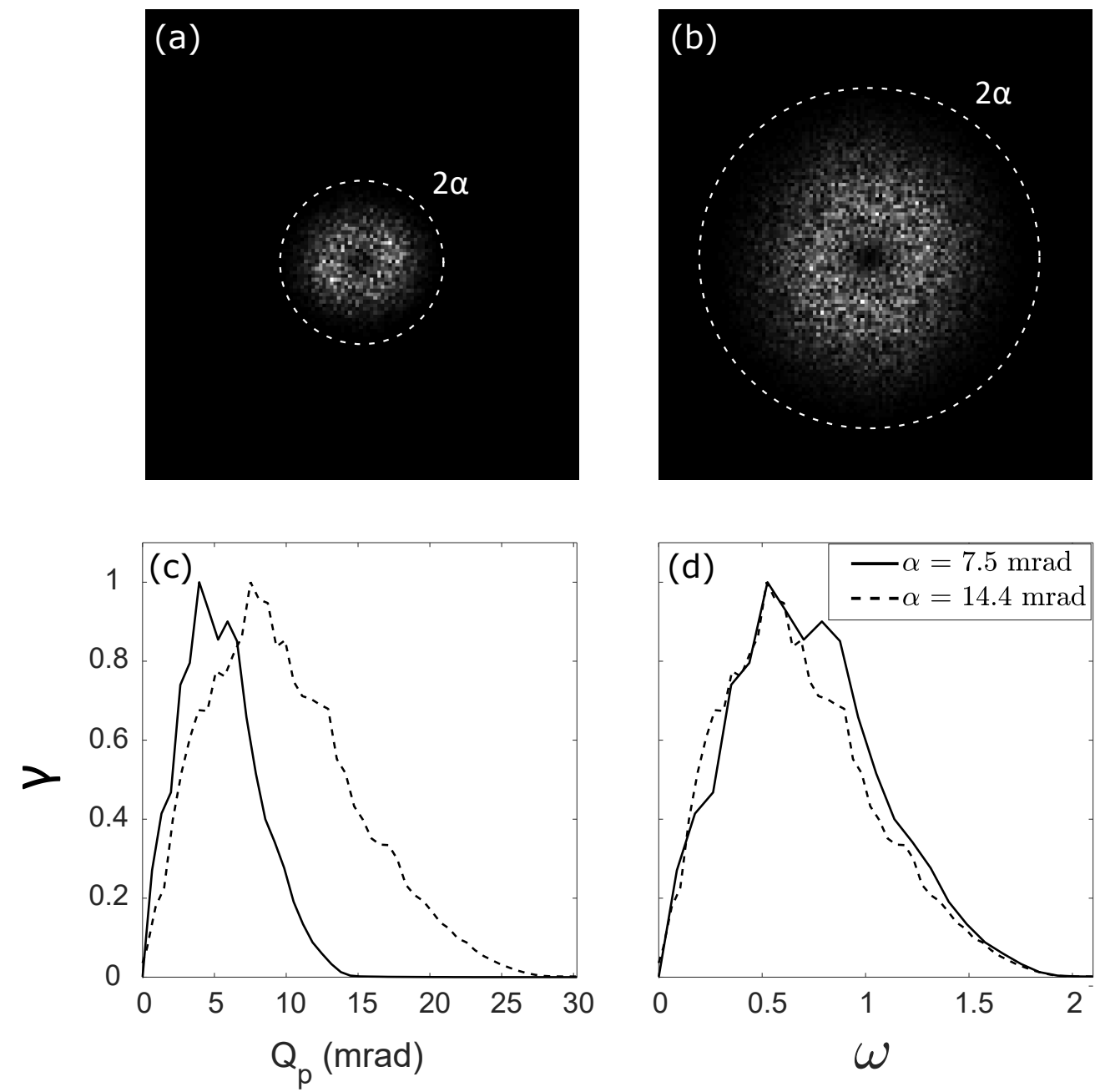

Figure 8: Power spectra of the SSB phase reconstructions from amorphous carbon for (a) $\alpha=7.5 \mathrm{mrad}$ and (b) $\alpha=14.4 \mathrm{mrad}$. The dashed circles correspond to the $2 \alpha$-limit beyond which no spatial frequencies are transferred to the reconstructed phase. (c) - (d) Azimuthal average of (a) (solid lines) and (b) (dashed lines) with respect to (c) $Q_{p}$ and (d) $\omega$.

trate the effects of the PCTFs for SSB and ePIE methods, we consider the aberration-corrected SSB phase reconstruction of a functionalised carbon nanotube (FCNT) shown in Figure 11(a) (previously presented in [33]). The data was acquired at an acceleration voltage of $80 \mathrm{kV}$, a convergence semi-angle of $22 \mathrm{mrad}$ and an electron dose of $\sim 2.7 \times 10^{4}$ e $\AA^{-2}$. Although the phase reconstruction shows atomic resolution, another feature is noticeable: a dark fringe which outlines the FCNT. This fringe is most likely a direct result of the suppression of low and high spatial frequencies. To demonstrate this, Figure 11(b) shows the phase reconstruction of the same FCNT using the ePIE algorithm [26]. There is sufficient signal outside the $\mathrm{BF}$ disc for ePIE to reconstruct the FCNT at a resolution beyond the $\omega=2$ limit of SSB ptychography. Furthermore, the broad transfer window of the ePIE method does not transfer edge artefacts to the image plane as for SSB ptychography. However, a slowly varying phase ramp in the vacuum is present across the entire field of view. This is possibly due to the amplification of low frequency noise or experimental effects such as the BF disc shifting slightly due to the probe scan system. This phase ramp can be avoided by applying a band-pass filter, such as that intrinsic to SSB ptychography, to the Fourier transmission function $\Psi\left(\mathbf{Q}_{\mathbf{p}}\right)$. The result of applying such a filter, shown in Figure 11(c), is a phase reconstruction that is almost identical to that shown in Figure 11(a): no slowly varying phase ramp, but a rapid phase dip around the FCNT walls.

Although both SSB and ePIE methods were performed using the same experimental data, the reconstructed complex transmission functions were not identical. This difference depends on how much of the 4-D STEM data set is used by each algorithm, and how each reconstruction algorithm is performed to produce a complex transmission function. For example, the SSB method only uses the BF disc in the reconstruction process, while the ePIE method also uses information from beyond the BF disc, which can lead to resolution improvement if this is needed [28]. However, for very 

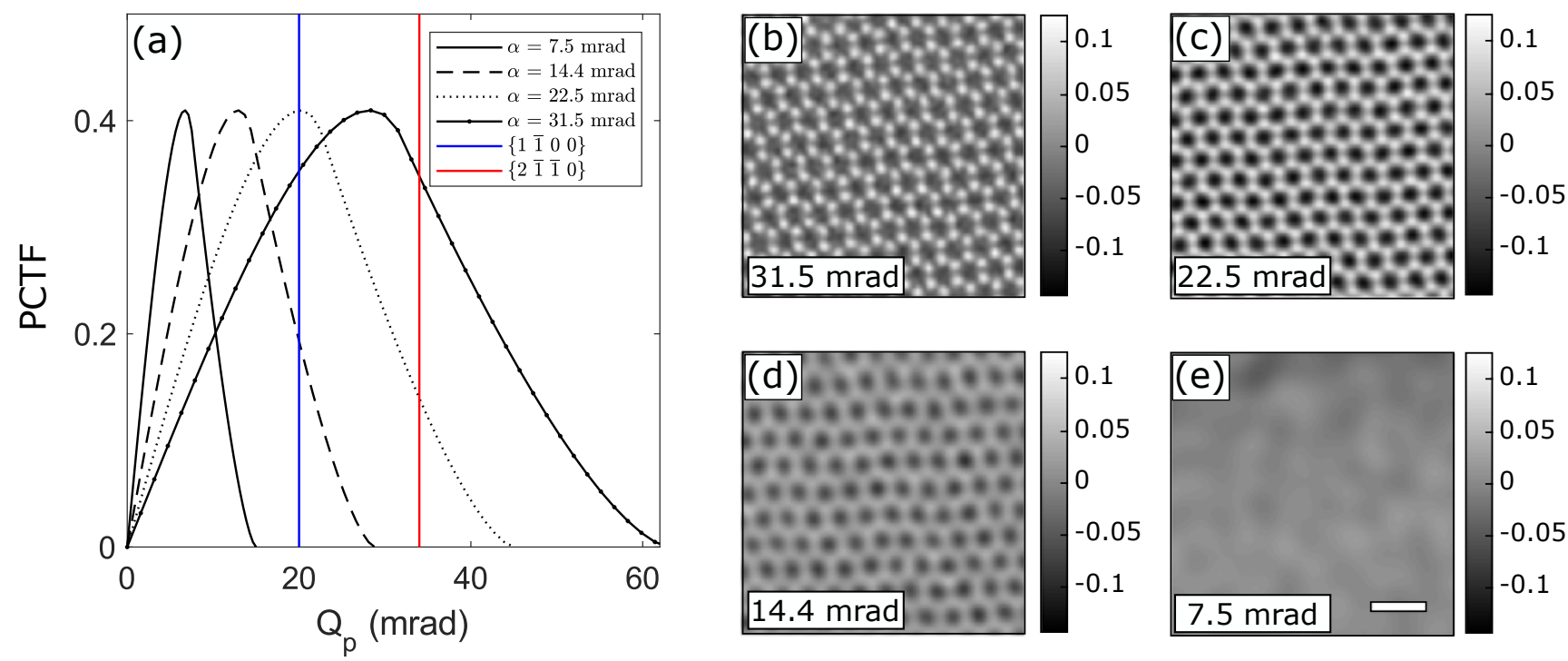

Figure 9: (a) PCTF for convergence semi-angles of $31.5 \mathrm{mrad}, 22.5 \mathrm{mrad}, 14.4 \mathrm{mrad}$, and $7.5 \mathrm{mrad}$ calculated using (8). The red and blue vertical lines represent reciprocal space locations of the $\{1 \overline{1} 00\}$ and $\{2 \overline{1} \overline{1} 0\}$ families of reflections. The phase reconstructions of experimental graphene data for each of the convergence semi-angles in (a) are shown in (b)-(e). Scale bar: $5 \AA$. Greyscale: phase (radians).

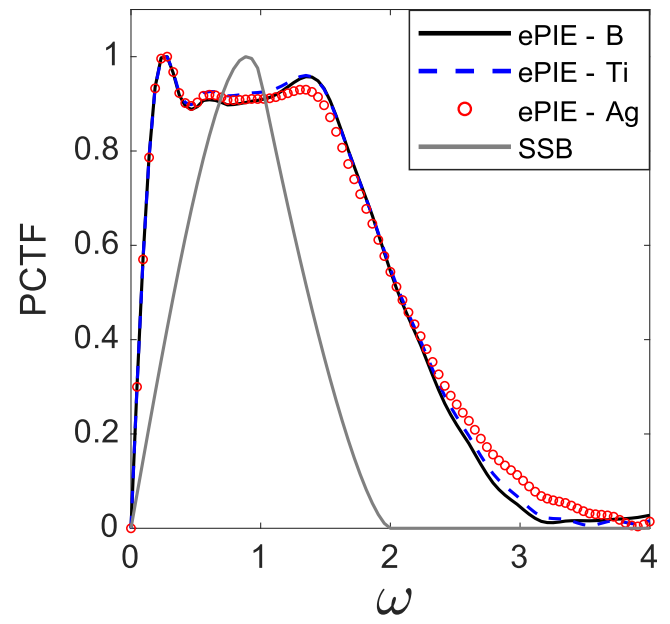

Figure 10: Plots of the normalised PCTF for the ePIE method, generated using isolated atom simulations. The PCTF for the SSB method is included for reference (grey line).

thin samples or at low electron doses, the super-resolution capabilities are reduced and the ePIE resolution limit converges to the $2 \alpha$ limit for SSB ptychography [20]. Furthermore, the PCTF of SSB and ePIE are strikingly different at low and high spatial frequencies. The ptychographic imaging process can be regarded as being performed in silico and is therefore algorithm dependent, unlike conventional imaging modes where the PCTF is defined by the microscope optics. Therefore, we can choose an alternative, target, transfer function, and decide on the PCTF to use, as in Figure 11(c). Other 4-D STEM phase imaging methods, such as iCOM and iDPC, will have their own intrinsic PCTFs, but are also sometimes combined with a Fourier filtering step to give a more desirable PCTF [19].

One of the most logical approaches to obtaining a suitable target transfer function is to account for the noise present in the 4-D STEM data. For scintillator-based detectors such as CCD and CMOS devices, the main contributions of noise are the discrete nature of charge carriers (statistical/shot noise), the thermal generation of electronhole pairs (thermal noise) and the detector readout process (readout noise). However, the contributions of thermal and readout noise are significantly reduced by using modern direct electron detectors [47]. For example, the combination of faster acquisition speeds $\left(10^{3}\right.$ frames per second) and the omission of coupling optics significantly reduces the readout noise $[48,49,50]$. Furthermore, detector systems are often cooled to temperatures below $0^{\circ} \mathrm{C}$ to reduce the thermal generation of charge carriers [48, 49], and threshold voltages can be set to neglect any residual dark current [50]. In such cases, the noise is predominantly statistical and can be modelled by a Poisson process which is well-approximated by a Gaussian/normal distribution at high electron doses [51]. The measurement at each $\left(\mathbf{K}_{\mathbf{f}}, \mathbf{R}_{\mathbf{p}}\right)$ coordinate is independent of any other measurement in the 4-D STEM data set. As such, the noise at each pixel is uncorrelated with detector plane position $\mathbf{K}_{\mathbf{f}}$ or probe position $\mathbf{R}_{\mathbf{p}}$. Therefore, it can be concluded that the noise distribution across a 4-D STEM data set acquired with a state-of-the-art detector is white noise, i.e. it is equally distributed across all spatial frequencies $\mathbf{Q}_{\mathbf{p}}$ and all real-space position vectors $\mathbf{r}$.

To examine the use of target transfer functions for SSB ptychography, three separate transfer functions were applied to ptychographic reconstructions: 1) the intrinsic PCTF (Figure 12(a)), 2) a flat transfer function (Figure 12(b)) and 3) a noise-normalised transfer function (Figure 12(c)). All 

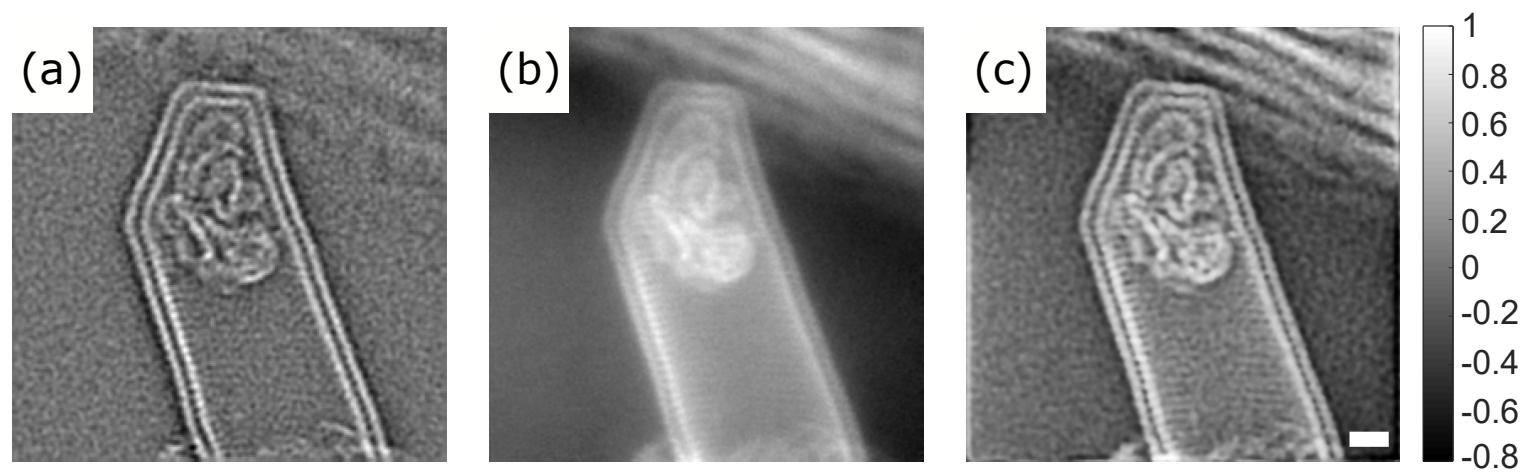

Figure 11: Aberration-corrected ptychographic reconstructions of a FCNT using (a) the SSB method, (b) standard ePIE and (c) SSB band-pass filtered ePIE. Scale bar: $5 \AA$. The maximum phase in each reconstruction has been normalised to 1 for consistency. Greyscale: normalised phase.

three transfer functions are described in more detail below. The ptychographic reconstructions were performed on a simulated carbon atom and two experimental graphene data sets. The same conditions were used in each case $\left(\mathrm{V}_{\text {acc }}=\right.$ $80 \mathrm{kV}, \alpha=22.5 \mathrm{mrad},\left|\Delta \mathbf{R}_{\mathbf{p}}\right|=0.13 \AA$ ). The 4-D matrix for the carbon atoms was simulated using the MULTEM code [46]. For each graphene data set, the residual aberrations were corrected by post-processing. Isolated carbon atom simulations were reconstructed using an electron dose identical to the experiment, the results of which are plotted in the insets to the graphene reconstructions throughout Figure 12.

\section{Intrinsic transfer function}

In conventional SSB ptychography, the boundaries of the reconstructed phase of the carbon has dips due to the bandpass nature of the PCTF (Figures 6 and 12). This manifests itself as a dark halo around the carbon atom simulation in Figure 12(d). Furthermore, the atomic spacings of graphene (i.e. the $\{2 \overline{1} \overline{1} 0\}$ reflections) are poorly resolved using a probe convergence semi-angle of $22.5 \mathrm{mrad}$, as shown in Figures 12(g) and (j).

\section{Flat transfer function}

Applying a target transfer function to the carbon atom such that spatial frequencies up to $2 \alpha / \lambda$ are transferred with equal weight, we obtain the phase reconstruction in Figure 12(e). This is the equivalent to normalising the contrast transfer to the area of the DO region at each $\mathbf{Q}_{\mathbf{p}}$ value. A Hann window roll-off function was applied at low and high values of $\mathbf{Q}_{\mathbf{p}}$ to reduce artefacts. The implementation of this target transfer function removes the negative phase dip visible in Figure 12(d). For the case of graphene shown in Figure 12(h), applying a flat transfer function resolves the atomic spacings with higher contrast. The flat transfer function enables a broader range of information transfer at high electron doses (i.e. $\sim 10^{6} \mathrm{e} \AA^{-2}$ ). However, if a flat transfer function is applied to lower electron doses (i.e. $\sim 10^{5}$ e $\AA^{-2}$ ), noise dominates the reconstructions, manifested as high-frequency ringing in Figure 12(k).

\section{Noise-normalised transfer function}

An alternative approach would be to synthesize a target transfer function which is normalised to the noise distribution. The SSB method only utilises parts of the detector plane which contain information about a particular sample spatial frequency. Other regions of the detector plane, which contain only noise, are rejected. The SSB approach can be regarded as being closely related to DPC, but with the detector adjusting its shape as a function of spatial frequency to optimise the signal-to-noise. As previously discussed by Seki et al. [51], the integration of statistical noise over the DO regions leads to a total noise level for a particular spatial frequency that is given by the square-root of the DO area, i.e. the square root of the intrinsic SSB PCTF. Producing a transfer function that has a flat noise transfer is therefore implemented in practise by using the square root of the intrinsic PCTF as the target transfer function. This is the equivalent of dividing the integrated SSB signal by the square root of the intrinsic PCTF (i.e. the shot noise) at each spatial frequency. Such a target transfer function has the advantage of mitigating the artefacts of the intrinsic SSB PCTF (i.e. the carbon atom reconstruction shown in Figure 12(f)), while keeping the noise at a constant level across all spatial frequencies. If this transfer function is applied to the graphene reconstructions, as shown in Figures 12(i) and (l), the $\{2 \overline{1} \overline{1} 0\}$ reflections are resolved with high contrast and the amplification of noise is avoided.

\section{Reducing noise by avoiding a deconvolution step}

As discussed in Section 2, the two most-common methods of non-iterative focused-probe ptychography are the SSB and WDD methods. Although both techniques effectively provide the same result for a weak-phase-object, there are subtle differences which should affect the choice of technique. The SSB method assumes a weak-phase-object as the sample, whereas for 'stronger' phase-objects, the WDD method is more applicable [25, 33, 34]. However, due to the nature of the Wiener deconvolution, one must exercise cau- 
Intrinsic
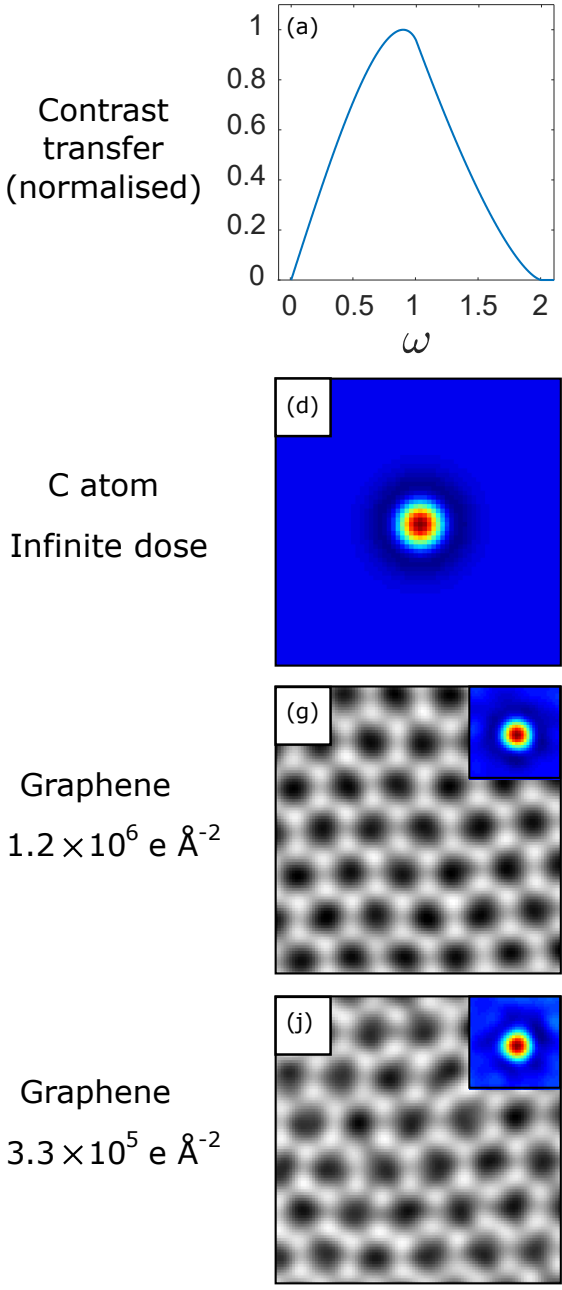

Flat
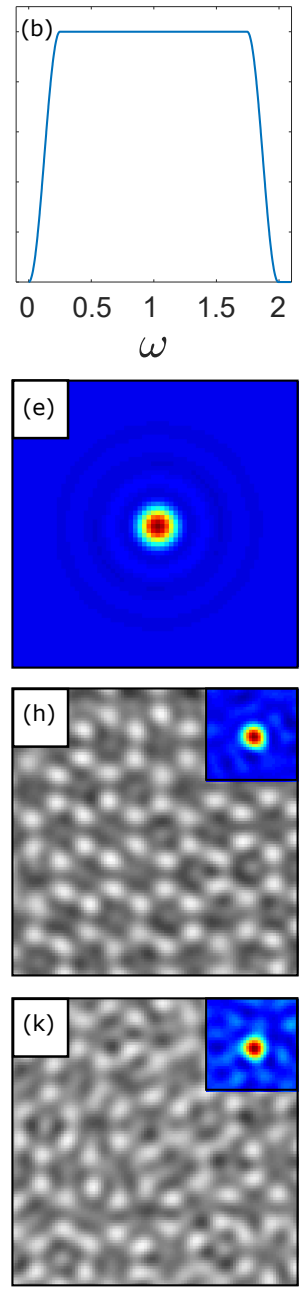

Noise-normalised
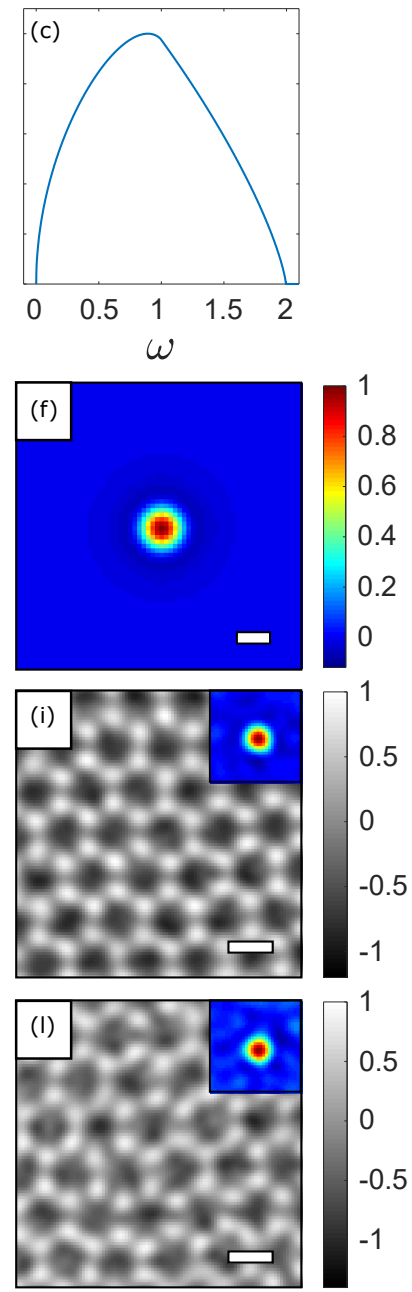

Figure 12: Comparison of transfer functions used in SSB ptychography. (a) Intrinsic, (b) flat and (c) noise-normalised contrast transfer functions. (d)-(I) SSB phase reconstructions using the transfer functions plotted in (a)-(c). The maximum phase in each reconstruction has been normalised to 1 for consistency. Colour bar: normalised phase. (d)-(f) Simulated carbon atom using infinite dose. Scale bar: $1 \AA$. (g)-(i) Experimental phase reconstructions of graphene (aberration-corrected) using a dose of $1.2 \times 10^{6}$ e $\AA^{-2}$. Scale bar: $2 \AA$. (j)(I) Experimental phase reconstructions of graphene (aberration-corrected) using a dose of $3.3 \times 10^{5}$ e $\AA^{-2}$. Scale bar: $2 \AA$. Insets of $(\mathrm{g})-(\mathrm{I})$ : phase reconstructions of carbon atom simulations at the same dose used for the experimental graphene reconstructions.

tion when applying the WDD method at low electron dose [25]. In particular, the order of $\varepsilon_{\text {rat }}$ (defined in (5)) affects how much signal and noise is suppressed in the reconstruction. To demonstrate this, Figure 13 shows a phase reconstruction for an aluminosilicate zeolite (ZSM-5) at an electron dose of 200 e $\AA^{-2}$ using (a) the SSB method and (b)-(d) the WDD method for different values of $\varepsilon_{\text {rat }}\left(\mathrm{V}_{\mathrm{acc}}=300 \mathrm{kV}\right.$, $\alpha=7.6 \mathrm{mrad})$. The SSB reconstruction, shown in Figure 13(a), is obtained without performing a deconvolution step, and thus avoids noise amplification. In contrast, reconstructing the transmission function using the WDD method for small values of $\varepsilon_{\text {rat }}(0.01-0.1)$ as used in the literature $[32,33]$ can give rise to noise amplification, as seen in Figures 13(b) and (c). Using $\varepsilon_{\text {rat }}=1$ as shown in Figures 13(d) effectively suppresses the noise, however, such a large filter parameter may also suppress useful sample information. To improve the robustness to noise for low-dose focusedprobe electron ptychography, it is therefore advisable to use the SSB method where applicable. Furthermore, the application of an aberration correction step to the SSB method, as discussed in Section 2, has removed the need to fine-tune the aberrations before data acquisition, and thus increases the throughput and dose-efficiency of SSB ptychography.

\section{Conclusions}

In this paper, the contrast transfer and noise properties of electron ptychography have been explored using the- 


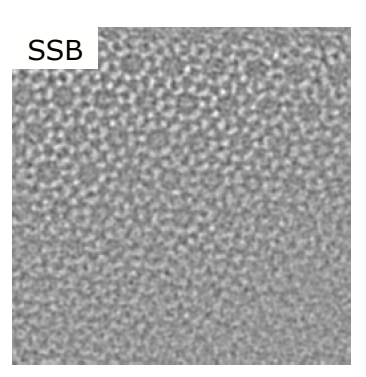

(a)

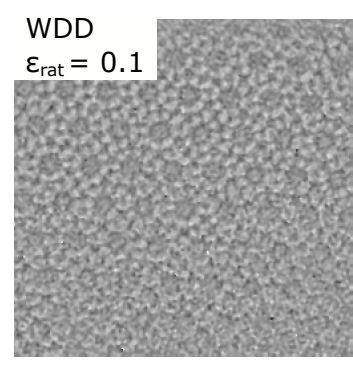

(c)

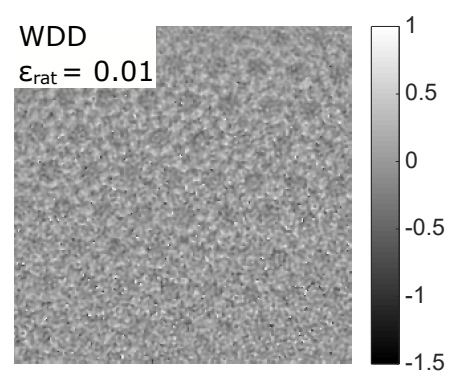

(b)

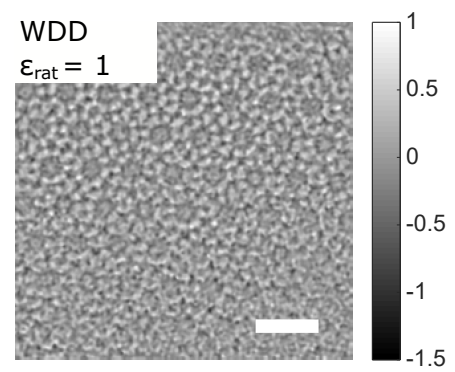

(d)
Figure 13: Comparison of phase reconstruction methods for ZSM-5 at an electron dose of 200 e $\AA^{-2}$. (a) SSB phase reconstruction. (b)-(d) WDD phase reconstructions for $\varepsilon_{\text {rat }}=0.01$, 0.1 and 1 , respectively. Scale bar: $2 \mathrm{~nm}$. The maximum phase in each reconstruction has been normalised to 1 for consistency.

ory, simulations and experiments. It was demonstrated that, by choosing a suitable probe convergence semi-angle, target transfer function and reconstruction algorithm, the dose needed to obtain high-SNR ptychographic reconstructions can be minimised. This is particularly important for increasing the contrast of light elements in phase reconstructions, and for imaging beam-sensitive materials using a sufficiently low electron dose to avoid beam damage.

Electron ptychography is growing in popularity since the introduction of direct electron detectors. High-contrast ptychographic imaging of light elements has become routinely achievable, and boasts a greater dose-efficiency than many common STEM imaging techniques. The absolute contrast transfer in electron ptychography will be somewhat dependent on the dose, although the relative contrast transfer will be dependent upon the aperture function (aperture size and wave aberrations). Even at high electron doses, a poorly chosen aperture will provide a reconstruction with a high noise level. The transfer function is also strongly dependent on the post-processing algorithm used - each 4-D STEM phase reconstruction technique possesses its own intrinsic transfer function. A target transfer function can be defined for SSB ptychography which transfers a broader range of spatial frequencies than the intrinsic transfer function, while avoiding noise amplification. In practise, this is achieved by normalising the contrast transfer to the signal-to-noise ratio. There are alternative methods to dealing with low-dose imaging, such as Bayesian inference, although some prior information about the sample is needed for this approach [52, 53]. Ptychography has been viewed as a potential technique for
4-D STEM imaging of biological materials at low electron doses $[20,54]$. The speed of 4-D STEM data acquisition ( $\sim 10^{3}$ frames per second) is currently a limiting factor for low-dose applications, but advances such as ultra-fast detectors [55] and binary data acquisition [27] should enable 4-D STEM at ADF imaging speeds over the next decade.

\section{Acknowledgements}

This research was supported by the Henry Royce Institute, the EPSRC (Grant No. EP/M010708/1) and JEOL (UK) Ltd. COL and PDN are grateful to PNDetector for their support in using the JEOL 4DCanvas pixelated detector. We thank Diamond Light Source for access and support in the use of the electron Physical Science Imaging Centre (Instrument E02, Proposal Nos. MG20431 and MG22317) that contributed to the results presented here.

\section{Data availability}

The data sets generated and analysed during this study are available from the corresponding author on reasonable request.

\section{References}

[1] R. F. Egerton. Radiation damage to organic and inorganic specimens in the TEM. Micron, 119:72-87, 2019. doi:10.1016/j.micron.2019.01.005.

[2] O. Scherzer. The theoretical resolution limit of the electron microscope. Journal of Applied Physics, 20(1):20-29, 1949. doi:10.1063/1.1698233.

[3] M. A. O'Keefe, E. C. Nelson, Y. C. Wang, and A. Thust. Sub-ångström resolution of atomistic structures below 0.8 å. Philosophical Magazine B, 81(11):1861-1878, 2001. doi:10.1080/13642810108223123.

[4] S. J. Haigh, H. Sawada, and A. I. Kirkland. Atomic Structure Imaging Beyond Conventional Resolution Limits in the Transmission Electron Microscope. Physical Review Letters, 103(12):126101, 2009. doi:10.1103/PhysRevLett.103.126101.

[5] S. J. Haigh, B. Jiang, D. Alloyeau, C. Kisielowski, and A. I. Kirkland. Recording low and high spatial frequencies in exit wave reconstructions. Ultramicroscopy, 133:26-34, 2013. doi:10.1016/j.ultramic.2013.04.012.

[6] S. J. Haigh. Super Resolution Tilt Series Exit Wave Restoration from Aberration Corrected Images. DPhil Thesis, University of Oxford, 2008.

[7] C. Huang. Ultra High Resolution Imaging of Radiation-Sensitive Materials. PhD thesis, University of Oxford, 2016.

[8] J. M. Cowley. Image contrast in a transmission scanning electron microscope. Applied Physics Letters, 15(2):58-59, 1969. doi:10.1063/1.1652901.

[9] A. V. Crewe. Scanning electron microscopes: is high resolution possible? Science, 154(3750):729-738, 1966. doi:10.1126/science.154.3750.729.

[10] A. V. Crewe. Visibility of single atoms. Science, 168(3937):13381340, 1970. doi:10.1126/science.168.3937.1338.

[11] P. D. Nellist and S. J. Pennycook. The principles and interpretation of annular dark-field Z-contrast imaging. In Advances in Imaging and Electron Physics, volume 113, pages 147-203. Elsevier, 2000. doi:10.1016/S1076-5670(00)80013-0.

[12] G. Black and E. H. Linfoot. Spherical aberration and the information content of optical images. Proceedings of the Royal Society of London. Series A. Mathematical and Physical Sciences, 239:522-540, 1957. doi:10.1098/rspa.1957.0059. 
[13] L. Jones and P.D. Nellist. Three-dimensional optical transfer functions in the aberration-corrected scanning transmission electron microscope. Journal of Microscopy, 254(2):47-64, 2014. doi:10.1111/jmi.12117.

[14] N. H. Dekkers and H. De Lang. Differential phase contrast in a STEM. Optik, 41(4):452-456, 1974.

[15] E. M. Waddell and J. N. Chapman. Linear imaging of strong phase objects using asymmetrical detectors in STEM. Optik, 54(2):83-96, 1979.

[16] K. Müller, F. F. Krause, A. Béché, M. Schowalter, V. Galioit, S. Löffler, J. Verbeeck, J. Zweck, P. Schattschneider, and A. Rosenauer. Atomic electric fields revealed by a quantum mechanical approach to electron picodiffraction. Nature Communications, 5(1):1-8, 2014. doi:10.1038/ncomms6653.

[17] N. Shibata, S. D. Findlay, Y. Kohno, H. Sawada, Y. Kondo, and Y. Ikuhara. Differential phase-contrast microscopy at atomic resolution. Nature Physics, 8(8):611-615, 2012. doi:10.1038/nphys2337.

[18] I. Lazić, E. G. T. Bosch, and S. Lazar. Phase contrast STEM for thin samples: Integrated differential phase contrast. Ultramicroscopy, 160:265-280, 2016. doi:10.1016/j.ultramic.2015.10.011.

[19] E. Yücelen, I. Lazić, and E. G. T. Bosch. Phase contrast scanning transmission electron microscopy imaging of light and heavy atoms at the limit of contrast and resolution. Scientific Reports, 8(1):1-10, 2018. doi:10.1038/s41598-018-20377-2.

[20] L. Zhou, J. Song, J. S. Kim, X. Pei, C. Huang, M. Boyce, L. Mendonça, D. Clare, A. Siebert, C. S. Allen, et al. Low-dose phase retrieval of biological specimens using cryo-electron ptychography. Nature Communications, 11:2994, 2020. doi:10.1038/s41467-02016391-6.

[21] W. Hoppe. Beugung im inhomogenen primärstrahlwellenfeld. I. Prinzip einer phasenmessung von elektronenbeungungsinterferenzen. Acta Crystallographica Section A: Crystal Physics, Diffraction, Theoretical and General Crystallography, 25(4):495-501, 1969. doi: $10.1107 /$ S0567739469001045.

[22] W. Hoppe and G. Strube. Beugung im inhomogenen primärstrahlwellenfeld. II. Lichtoptische analogieversuche zur phasenmessung von gitterinterferenzen. Acta Crystallographica Section A: Crystal Physics, Diffraction, Theoretical and General Crystallography, 25(4):502-507, 1969. doi:10.1107/S0567739469001057.

[23] W. Hoppe. Beugung im inhomogenen primärstrahlwellenfeld. III. Amplituden-und phasenbestimmung bei unperiodischen objekten. Acta Crystallographica Section A: Crystal Physics, Diffraction, Theoretical and General Crystallography, 25(4):508-514, 1969. doi: 10.1107/S0567739469001069.

[24] J. M. Rodenburg, B. C. McCallum, and P. D. Nellist. Experimental tests on double-resolution coherent imaging via STEM. Ultramicroscopy, 48(3):304-314, 1993. doi:10.1016/0304-3991(93)901057.

[25] J. M. Rodenburg and R. H. T. Bates. The theory of superresolution electron microscopy via Wigner-distribution deconvolution. Philosophical Transactions of the Royal Society of London. Series A: Physical and Engineering Sciences, 339(1655):521-553, 1992. doi:10.1098/rsta.1992.0050.

[26] A. M. Maiden and J. M. Rodenburg. An improved ptychographical phase retrieval algorithm for diffractive imaging. Ultramicroscopy, 109(10):1256-1262, 2009. doi:10.1016/j.ultramic.2009.05.012.

[27] C. M. O’Leary, C. S. Allen, C. Huang, J. S. Kim, E. Liberti, P. D. Nellist, and A. I. Kirkland. Phase reconstruction using fast binary 4D STEM data. Applied Physics Letters, 116(112):124101, 2020. doi: 10.1063/1.5143213.

[28] Y. Jiang, Z. Chen, Y. Han, P. Deb, H. Gao, S. Xie, P. Purohit, M. W. Tate, J. Park, S. M. Gruner, et al. Electron ptychography of 2D materials to deep sub-ångström resolution. Nature, 559(7714):343-349, 2018. doi:10.1038/s41586-018-0298-5.

[29] J. Song, C. S. Allen, S. Gao, C. Huang, H. Sawada, X. Pan, J. Warner, P. Wang, and A. I. Kirkland. Atomic resolution defocused electron ptychography at low dose with a fast, direct electron detector. Scientific Reports, 9:3919, 2019. doi:10.1038/s41598-019-40413-z.
[30] Z. Chen, M. Odstrcil, Y. Jiang, Y. Han, M. H. Chiu, L. J. Li, and D. A. Muller. Mixed-state electron ptychography enables sub-angstrom resolution imaging with picometer precision at low dose. Nature Communications, 11:2773, 2020. doi:10.1038/s41467-020-16688-6.

[31] P. M. Pelz, R. Bücker, G. Ramm, H. Venugopal, G. Kassier, D. Eggert, P. H. Lu, R. E. Dunin-Borkowski, and R. J. D. Miller. Electron Ptychography of Single Biological Macromolecules. Microscopy and Microanalysis, 25(S2):72-73, 2019. doi:10.1017/S1431927619001090.

[32] H. Yang, I. MacLaren, L. Jones, G. T. Martinez, M. Simson, M. Huth, H. Ryll, H. Soltau, R. Sagawa, Y. Kondo, et al. Electron ptychographic phase imaging of light elements in crystalline materials using Wigner distribution deconvolution. Ultramicroscopy, 180:173-179, 2017. doi:10.1016/j.ultramic.2017.02.006.

[33] H. Yang, R. N. Rutte, L. Jones, M. Simson, R. Sagawa, H. Ryll, M. Huth, T. J. Pennycook, M. L. H. Green, H. Soltau, et al. Simultaneous atomic-resolution electron ptychography and Z-contrast imaging of light and heavy elements in complex nanostructures. Nature Communications, 7(1):1-8, 2016. doi:10.1038/ncomms 12532 .

[34] J. G. Lozano, G. T. Martinez, L. Jin, P. D. Nellist, and P. G. Bruce. Low-Dose Aberration-Free Imaging of Li-Rich Cathode Materials at Various States of Charge Using Electron Ptychography. Nano Letters, 18(11):6850-6855, 2018. doi:10.1021/acs.nanolett.8b02718.

[35] T. Chen, I. Ellis, T. J. N. Hooper, E. Liberti, L. Ye, B. T. W. Lo, C. O'Leary, A. A. Sheader, G. T. Martinez, L. Jones, et al. Interstitial Boron Atoms in the Palladium Lattice of an Industrial Type of Nanocatalyst: Properties and Structural Modifications. Journal of the American Chemical Society, 141(50):19616-19624, 2019. doi:10.1021/jacs.9b06120.

[36] K. Ishizuka. Contrast transfer of crystal images in TEM. Ultramicroscopy, 5(1-3):55-65, 1980. doi:10.1016/0304-3991(80)90011-X.

[37] T. J. Pennycook, A. R. Lupini, H. Yang, M. F. Murfitt, L. Jones, and P. D. Nellist. Efficient phase contrast imaging in STEM using a pixelated detector. Part 1: Experimental demonstration at atomic resolution. Ultramicroscopy, 151:160-167, 2015. doi:10.1016/j.ultramic.2014.09.013.

[38] P. D. Nellist. Scanning Transmission Electron Microscopy. In Springer Handbook of Microscopy, pages 49-99. Springer International Publishing, 2019. ISBN 978-3-030-00069-1. doi:10.1007/9783-030-00069-1_2.

[39] T. J. Pennycook, G. T. Martinez, P. D. Nellist, and J. C. Meyer. High dose efficiency atomic resolution imaging via electron ptychography. Ultramicroscopy, 196:131-135, 2019. doi:10.1016/j.ultramic.2018.10.005.

[40] J. M. Rodenburg and H. M. L. Faulkner. A phase retrieval algorithm for shifting illumination. Applied Physics Letters, 85(20):4795-4797, 2004. doi:10.1063/1.1823034.

[41] P. Wang, F. Zhang, S. Gao, M. Zhang, and A. I. Kirkland. Electron ptychographic diffractive imaging of boron atoms in $\mathrm{LaB}_{6}$ crystals. Scientific Reports, 7(1):1-8, 2017. doi:10.1038/s41598-017-02778$\mathrm{x}$.

[42] H. Yang, T. J. Pennycook, and P. D. Nellist. Efficient phase contrast imaging in STEM using a pixelated detector. Part II: Optimisation of imaging conditions. Ultramicroscopy, 151:232-239, 2015. doi:10.1016/j.ultramic.2014.10.013.

[43] H. Yang, P. Ercius, P. D. Nellist, and C. Ophus. Enhanced phase contrast transfer using ptychography combined with a pre-specimen phase plate in a scanning transmission electron microscope. Ultramicroscopy, 171:117-125, 2016. doi:10.1016/j.ultramic.2016.09.002.

[44] F. Thon. Zur Defokussierungsabhängigkeit des Phasenkontrastes bei der elektronenmikroskopischen Abbildung. Zeitschrift für Naturforschung A, 21(4):476-478, 1966. doi:10.1515/zna-1966-0417.

[45] S. E. Zeltmann, A. Müller, K. C. Bustillo, B. Savitzky, L. Hughes, A. M. Minor, and C. Ophus. Patterned probes for high precision 4DSTEM bragg measurements. Ultramicroscopy, 209:112890, 2020. doi:10.1016/j.ultramic.2019.112890.

[46] I. Lobato and D. Van Dyck. MULTEM: A new multislice program to perform accurate and fast electron diffraction and imaging simulations 
using Graphics Processing Units with CUDA. Ultramicroscopy, 156: 9-17, 2015. doi:10.1016/j.ultramic.2015.04.016.

[47] R. Clough and A. I. Kirkland. Direct Digital Electron Detectors. In Advances in Imaging and Electron Physics, volume 198, pages 1-42. Elsevier, 2016. doi:10.1016/bs.aiep.2016.09.001.

[48] H. Ryll, M. Simson, R. Hartmann, P. Holl, M. Huth, S. Ihle, Y. Kondo, P. Kotula, A. Liebel, K. Müller-Caspary, et al. A pnCCD-based, fast direct single electron imaging camera for TEM and STEM. Journal of Instrumentation, 11(04):P04006, 2016. doi:10.1088/17480221/11/04/P04006.

[49] M. W. Tate, P. Purohit, D. Chamberlain, K. X. Nguyen, R. Hovden, C. S. Chang, P. Deb, E. Turgut, J. T. Heron, D. G. Schlom, et al. High Dynamic Range Pixel Array Detector for Scanning Transmission Electron Microscopy. Microscopy and Microanalysis, 22(1): 237-249, 2016. doi:https://doi.org/10.1017/S1431927615015664.

[50] J. A. Mir, R. Clough, R. MacInnes, C. Gough, R. Plackett, I. Shipsey, H. Sawada, I. MacLaren, R. Ballabriga, D. Maneuski, et al. Characterisation of the Medipix 3 detector for 60 and $80 \mathrm{keV}$ electrons. Ultramicroscopy, 182:44-53, 2017. doi:10.1016/j.ultramic.2017.06.010.

[51] T. Seki, Y. Ikuhara, and N. Shibata. Theoretical framework of statistical noise in scanning transmission electron microscopy. Ultramicroscopy, 193:118-125, 2018. doi:10.1016/j.ultramic.2018.06.014.

[52] J. Fatermans, A. J. den Dekker, K. Müller-Caspary, I. Lobato, C. M. O'Leary, P. D. Nellist, and S. Van Aert. Single atom detection from low contrast-to-noise ratio electron microscopy images. Physical Review Letters, 121(5):056101, 2018. doi:10.1103/PhysRevLett.121.056101.

[53] J. Fatermans, S. Van Aert, and A. J. den Dekker. The maximum a posteriori probability rule for atom column detection from HAADF STEM images. Ultramicroscopy, 201:81-91, 2019. doi:10.1016/j.ultramic.2019.02.003.

[54] P. M. Pelz, W. X. Qiu, R. Bücker, G. Kassier, and R. J. D. Miller. Low-dose cryo electron ptychography via non-convex Bayesian optimization. Scientific Reports, 7(1):1-13, 2017. doi:10.1038/s41598017-07488-y.

[55] J. Ciston, I. J. Johnson, B. R. Draney, P. Ercius, E. Fong, A. Goldschmidt, J. M. Joseph, J. R. Lee, A. Mueller, C. Ophus, et al. The 4D Camera: Very High Speed Electron Counting for 4DSTEM. Microscopy and Microanalysis, 25(S2):1930-1931, 2019. doi:10.1017/S1431927619010389. 